\begin{tabular}{|c|l|}
\hline Title & Quasisteady streaming with rarefaction effect induced by asymmetric sawtooth-like plane waves \\
\hline Author(s) & Y ano, Takeru; Inoue, Y oshinori \\
\hline Citation & $\begin{array}{l}\text { Physics of Fluids, 8(9), 2537-2551 } \\
\text { https://doi.org/L0.1063/1.869036 }\end{array}$ \\
\hline Issue Date & 1996-09 \\
\hline Doc URL & http://hdl.handle.net/2115/7387 \\
\hline Rights & Copyright $\odot$ 1996 A merican Institute of Physics \\
\hline Type & article \\
\hline File Information & PF8_9.pdf \\
\hline
\end{tabular}

Instructions for use 


\title{
Quasisteady streaming with rarefaction effect induced by asymmetric
sawtooth-like plane waves
}

\author{
Takeru Yano and Yoshinori Inoue \\ Department of Engineering Science, Faculty of Engineering, Hokkaido University, Sapporo 060, Japan
}

(Received 29 January 1996; accepted 29 May 1996)

The nonlinear plane acoustic wave emitted from a harmonically oscillating plate into an ideal gas of semi-infinite extent develops into a sawtooth-like wave, as long as the energy dissipation is negligibly small everywhere except for discontinuous shock fronts. The present authors have recently studied the strongly nonlinear propagation process and, in particular, numerically shown that, contrary to the result of the conventional weakly nonlinear theory, streaming (mean mass flow) due to shocks occurs in the direction of wave propagation, and thereby the gas near the plate is rarefied as time proceeds [J. Acoust. Soc. Am. 94, 1632 (1993)]. In this paper, the analysis of strongly nonlinear problem is advanced by extending the numerical computation up to about 190 periods of oscillation of plate, which is about three times longer than the previous one. It is demonstrated that, in the course of time, a quasisteady state is established, where a low-density and high-entropy region formed near the plate continues to grow at almost constant rate and the quasisteady streaming endures outside the region. Furthermore, the weakly nonlinear problem is analytically examined by a perturbation method up to $O\left(M^{3}\right)[M(\ll 1)$ is the acoustic Mach number]. The result shows that a sawtooth-like profile loses its symmetry in the second-order, and this causes weak streaming of $O\left(M^{2}\right)$. The decrease in density of the gas can be related to the accumulation of the third-order effects of production of entropy and generation of reflected wave at each shock front. (C) 1996 American Institute of Physics. [S1070-6631(96)02309-4]

\section{INTRODUCTION}

The nonlinear plane acoustic wave emitted from a harmonically oscillating plate into an ideal gas of semi-infinite extent propagates with its profile being distorted by the nonlinear effect (see Fig. 1). The nonlinear distortion leads to the formation of shock, and thereafter the wave profile progressively approaches a sawtooth-like one (see Fig. 2), as long as the energy dissipation due to viscosity and thermal conductivity is sufficiently small everywhere except for discontinuous shock fronts. According to the conventional first-order weakly nonlinear theory, the wave develops into the sawtooth wave of symmetric profile with no streaming (mean mass flow) [Fig. 2(a)], while a recent work ${ }^{1}$ for the strongly nonlinear case has shown that streaming is induced by an asymmetrical sawtooth-like wave [Fig. 2(b)] and streaming rarefies the gas near the oscillating plate. We shall investigate the streaming motion and the rarefaction phenomenon in the nonlinear propagation process of plane waves.

In a previous paper, ${ }^{1}$ the authors have studied a strongly nonlinear problem, which is characterized by the conditions

$$
M \equiv \frac{u_{0}}{c_{0}}=\frac{a \omega}{c_{0}}=O(1)
$$

and

$$
\operatorname{Re} \equiv \frac{(\gamma+1) c_{0} u_{0}}{\delta \omega}=\frac{(\gamma+1) c_{0} a}{\delta} \gg 1,
$$

where $M$ is the acoustic Mach number and Re is the acoustic Reynolds number $\left(c_{0}\right.$ is the speed of sound in an initial undisturbed gas, $a$ and $\omega$ are an amplitude and angular frequency of harmonic oscillation of the plate, $u_{0}=a \omega$ is the maximum speed of the oscillation, $\gamma$ is the ratio of specific heats for the ideal gas, and $\delta$ is the diffusivity of sound). ${ }^{2}$ The former condition, $M=O(1)$, means that the wave is a strongly nonlinear one, i.e., its profile is rapidly distorted and this leads to the shock formation near the plate. The latter, $\mathrm{Re} \gg 1$, means that we can regard the shock as a discontinuity, and may ignore the dissipation effect everywhere except for the discontinuity. ${ }^{3}$ In Ref. 1 , by using analytical and numerical methods, ${ }^{4,5}$ several strongly nonlinear phenomena have been found - the confluence of shocks, the generation of streaming, etc. They present striking contrasts to the established results of conventional weakly nonlinear theory based on the first-order solution in the case of

$$
M \ll 1 \quad \text { and } \operatorname{Re} \gg 1 \text {. }
$$

Among the strongly nonlinear phenomena, the generation of streaming and resulting rarefaction of the gas near the plate may be deserving special attention, because whether the wave motion can reach a steady state or not may strongly be affected by them. In this paper, focusing on streaming with rarefaction effect, we shall advance the analysis of the strongly nonlinear problem of $M=O(1)$ and $R e \gg 1$ and investigate the higher-order weakly nonlinear problem of $M \ll 1$ and $\operatorname{Re} \gg 1$.

The first-order problem in the weakly nonlinear case ${ }^{6,7}$ has been thoroughly examined for its fundamental importance, and the result is a matter of common knowledge in nonlinear acoustics now. ${ }^{8,9}$ The wave profile is hardly distorted in the near field (i.e., in the region whose distances from the plate are comparable with a wavelength $\left.2 \pi c_{0} / \omega\right)$, and the nonlinear effect manifests itself mainly in the far field (i.e., in the propagation process over large ranges compared with the wavelength). The wave profile at large distances approaches the so-called sawtooth wave, which has 


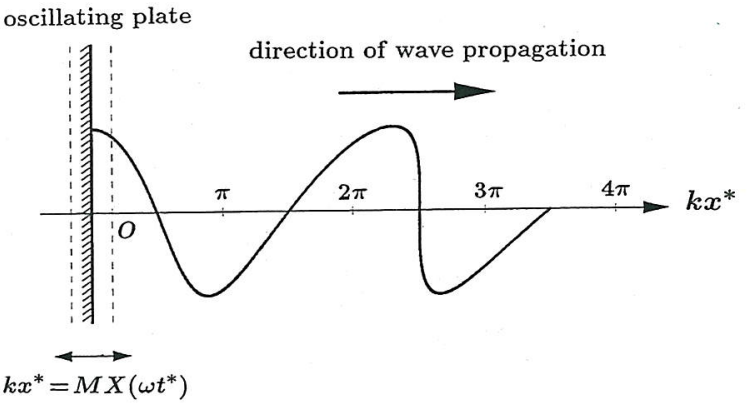

FIG. 1. Schematic of the model: generation of nonlinear plane acoustic wave by an oscillating infinite plate.

a symmetry between the compressive and rarefactive phases [Fig. 2(a)]. The phenomenon is in a steady state, i.e., it is periodically repeated with the period $2 \pi / \omega$. No streaming motion arises and a time average of density never changes. In the strongly nonlinear case, ${ }^{1}$ however, we have numerically shown that the wave of an asymmetrical sawtooth-like profile propagates with entraining the gas [Fig. 2(b)], i.e., streaming motion is excited, and that streaming rarefies the gas near the plate at least up to $t^{*}=120 \pi / \omega$, i.e., 60 periods of oscillation of plate ( $t^{*}$ is the time from the beginning of wave motion). Moreover, the entropy produced at shock fronts accumulates in the near field more and more as time goes by. Consequently, we have reached the conclusion that the phenomenon may not have the periodicity in the limit as $t^{*} \rightarrow \infty$, but may approach a quasisteady state. The similar streaming motions have been found in a spherical-wave problem $^{10}$ and in a piston problem. ${ }^{11}$ The conventional weakly nonlinear theory and the results in Ref. 1 are briefly reviewed in Sec. IIII.

However, since the previous numerical study was not carried far enough in time, there still remains a possibility that the drastic changes of density and entropy fields bring on some qualitative change of streaming motion. We there-
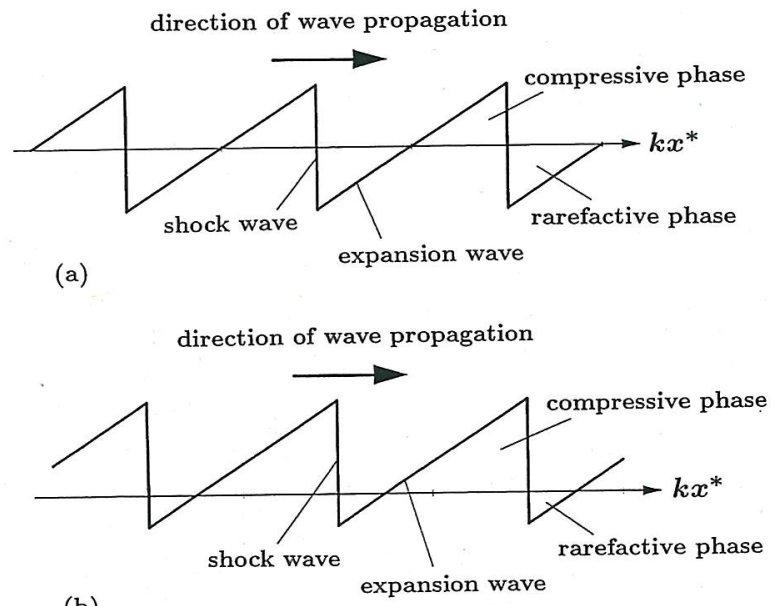

(b)

FIG. 2. Asymptotic profiles of the nonlinear waves radiated by a harmonic oscillation of plate. (a) The sawtooth wave (conventional first-order weakly nonlinear theory). (b) A sawtooth-like wave of asymmetric profile (strongly nonlinear case or higher-order weakly nonlinear theory). fore extend the numerical computation up to about 190 periods, and examine the long time evolution of wave field, using the same numerical method as in Ref. 1 (a highresolution upwind scheme ${ }^{4}$ with a flux-difference splitting ${ }^{5}$ ). Although the possibility is not completely removed even in the present study, the results reinforcing the conclusion in Ref. 1 can be obtained (see Sec. IV): a low-density and highentropy region formed near the plate continues to grow at almost constant rate, and outside the region streaming motion retains its quasisteadiness. Such a wave phenomenon may be regarded as being in a quasisteady state.

In order to deepen the understanding of the wave phenomenon involving streaming and rarefaction, some analytical study may also be required. An analytical treatment is possible if we restrict ourselves to the weakly nonlinear case of $M \ll 1$ and Re»1. In Secs. V and VI, we investigate the higher-order weakly nonlinear problem by a perturbation method in $M$ (i) to verify that streaming with rarefaction effect exists even in the case that the nonlinearity is weak, and (ii) to clarify the process of rarefaction and the relation between the decreasing density and the increasing entropy.

As shown by the second-order analysis in Sec. V, a sawtooth-like profile loses its symmetry in the second-order approximation, and this causes weak streaming of $O\left(M^{2}\right)$ in the far field beyond the shock formation distance (see Sec. $\mathrm{VB})$. The streaming carries the mass of the gas by an amount of $O\left(M^{2}\right)$ per unit time and per unit area, and thus the density decreases in a region where the streaming does not fully develop. Since the linear dimension of the region is of $O(1 / M)$, the decrease in density per unit time is of $O\left(M^{3}\right)$, and hence the determination of density distribution requires at least the third-order analysis.

The third-order analysis is carried out in Sec. VI, where we shall take into account the third-order effects of production of entropy and generation of reflected wave at each shock front by making use of the Rankine-Hugoniot relations. Lighthill ${ }^{12}$ has applied a similar technique for the investigation of the relation between the total energy behind a shock generated by a piston and the total entropy gain. The work of Lighthill has been extended to the two-dimensional steady supersonic flow past a thin symmetrical body by Phythian. ${ }^{13}$ In Sec. VI A, we shall derive a formula which determines the amplitude of reflected wave generated at any unsteady weak shock front. Our method of derivation is considerably simple compared with Lighthill's one. An equivalent formula has also been obtained by Morfey and Sparrow $^{14}$ in a problem where a compression wave connecting two different uniform states forms a shock and a reflected wave. The reflected wave and entropy production result in the negative density perturbation for $1<\gamma<5 / 3$ [we are concerned with air $(\gamma=1.4)]$. It is shown that the decrease in density is related to the reflected waves and the accumulation of entropy produced at a number of shock fronts (see Sec. VI B). The contribution of entropy is about eight times as large as that of reflected wave for $\gamma=1.4$. The analytical results agree with those obtained by the same numerical method as in Ref. 1 and Sec. IV for sufficiently small $M$. Comments on the validity of the analysis are given, in addition to the conclusions, in Sec. VII. 


\section{FORMULATION OF THE PROBLEM}

In order to formulate the problem, we shall introduce the nondimensional variables,

$$
t=\omega t^{*}, \quad x=\frac{x^{*} \omega}{c_{0}}, \quad u=\frac{u^{*}}{c_{0}}, \quad \rho=\frac{\rho^{*}}{\rho_{0}}, \quad p=\frac{p^{*}}{\rho_{0} c_{0}^{2}},
$$

where $x^{*}$ is the distance from an initial position of the plate, $u^{*}$ is the $x^{*}$ component of the fluid velocity, $\rho^{*}$ is the density of the gas, and $p^{*}$ is the pressure ( $\rho_{0}$ is an initial undisturbed density).

The condition Re $\gg 1$ allows neglecting the energy dissipation effect due to viscosity and thermal conductivity of the gas until a shock wave emerges in the wave field. Hence, at least until the time of shock formation, we can use the system of Euler equations for one-dimensional flow

$$
\begin{aligned}
& \frac{\partial \rho}{\partial t}+\frac{\partial(\rho u)}{\partial x}=0, \\
& \frac{\partial(\rho u)}{\partial t}+\frac{\partial\left(p+\rho u^{2}\right)}{\partial x}=0, \\
& \frac{\partial E_{\mathrm{t}}}{\partial t}+\frac{\partial\left[\left(E_{\mathrm{t}}+p\right) u\right]}{\partial x}=0,
\end{aligned}
$$

where $E_{\mathrm{t}}=\rho u^{2} / 2+p /(\gamma-1)$ is the normalized total energy of the ideal gas per unit volume. Once a shock is formed, the energy dissipation can no longer be ignored, which must in reality be produced at least at the shock front. As is generally known, however, a shock solution can be represented as a discontinuity ${ }^{15}$ in the system of equations (4)-(6), owing to the condition $\mathrm{Re} \gg 1$. We can thus employ this system even after the shock formation time. We shall obtain such a discontinuous solution under the initial and boundary conditions described below.

The gas is supposed to be uniform and at rest for $t \leqslant 0$. The initial conditions at $t=0$ are therefore

$$
u=0, \rho=1, p=\frac{1}{\gamma} \quad(x \geqslant 0) .
$$

The boundary condition on the plate is given by

$$
u=M X^{\prime}(t) \text { at } x=M X(t),
$$

where the prime denotes the differentiation with respect to the argument and $M X(t)$ is the instantaneous location of the plate (see Fig. 1); the function $X(t)$ is assumed to be

$$
X(t)=\left\{\begin{array}{l}
\cos t-1 \quad(t>0), \\
0 \quad(t \leqslant 0)
\end{array}\right.
$$

Up to the shock formation time, the wave is the simple wave, ${ }^{16}$ and the exact solution of system (4)-(6) satisfying the initial conditions (7) and the boundary condition (8) can be obtained by the method of characteristics ${ }^{1,17,18}$

$$
u=M X^{\prime}(\mu), \quad y=\mu-M X(\mu)-\beta M X^{\prime}(\mu)(t-\mu),
$$

where

$$
y=t-x,
$$

is the phase and

$$
\beta=\frac{\gamma+1}{2},
$$

is termed the parameter of nonlinearity in nonlinear acoustics. For a fixed $\mu$, the second of Eq. (10) represents a characteristic curve in the $(y, t)$ plane and then $\mu$ indicates the time when the characteristic is issued from the plate. The characteristic is a straight line along which the fluid velocity $u=M X^{\prime}(\mu)$ is constant and the slope $\mathrm{d} y / \mathrm{d} t$ of each characteristic is proportional to $u$. The undisturbed constant state corresponds to the region $y<0$, i.e., $\mu<0$. Once the function $u(y, t)$ has been found from Eq. (10), we can readily have other quantities as functions of $y$ and $t$ :

$$
p=\frac{1}{\gamma}\left(1+\frac{\gamma-1}{2} u\right)^{2 \gamma /(\gamma-1)}, \quad \rho=\left(1+\frac{\gamma-1}{2} u\right)^{2 /(\gamma-1)} .
$$

Here, the first of Eq. (13) represents the isentropic relation for the ideal gas and the second is derived from the fact that a Riemann invariant

$$
S=u-\frac{2 c}{\gamma-1},
$$

is equal to $-2 /(\gamma-1)$ everywhere $(c=\sqrt{\gamma p / \rho}$ is the nondimensional local speed of sound).

The velocity profile described by Eq. (10) is distorted as the wave propagates, and this waveform distortion eventually leads to the formation of shock at the smallest time $t_{\mathrm{s}}$ satisfying $\partial u / \partial y=\infty$. The time $t_{\mathrm{s}}$ and distance $x_{\mathrm{s}}$ of shock formation have been analyzed in Ref. 1, by using Eq. (10).

The assumption of a simple wave is invalid beyond the shock formation time $t_{s}$, because once a shock is formed the flow is no longer isentropic and the Riemann invariant $S$ is not constant across the shock. In the case of $M \ll 1$ and $R e \gg 1$, the production of entropy and jump of the Riemann invariant are of the third order of shock strength, i.e., they are of $O\left(M^{3}\right)$. Therefore, if we restrict ourselves to the second-order approximation of weakly nonlinear problem, we can neglect these third-order effects (Sec. V). In order to take into account these effects, we shall utilize the RankineHugoniot relations (Sec. VI). For the strongly nonlinear problem of $M=O(1)$ and $\mathrm{Re} \gg 1$, we shall employ a reliable numerical approach, a high-resolution upwind finite difference scheme ${ }^{4}$ (Sec. IV).

\section{SUMMARY OF PREVIOUS ANALYSES}

In this section, we shall shortly summarize the results from the conventional weakly nonlinear theory, and then review some of the results in Ref. 1 with focusing attention on streaming and closely related strongly nonlinear phenomena.

\section{A. Conventional weakly nonlinear theory $(M \ll 1, \operatorname{Re} \gg 1)$}

According to the conventional weakly nonlinear theory, the wave propagation is, up to the shock formation distance, described by the first-order solution, ${ }^{6,7}$ 


$$
v=X^{\prime}(\xi), \quad y=\xi-\sigma X^{\prime}(\xi) \quad\left(v \equiv \frac{u}{M}, \sigma \equiv \beta M x\right)
$$

where $v$ is a normalized fluid velocity, $\sigma$ is the distance from the plate normalized by the shock formation distance. For a fixed $\xi$, the second of Eq. (15) is the first-order approximation of characteristic curve, along which $v$ is constant (see Fig. 3).

At the nearest location where $\partial v / \partial y=\infty$, a shock is formed, so that solution (15) becomes invalid beyond there (the wave profile becomes triple valued). The shock formation distance $x_{\mathrm{s}}$ and shock formation time $t_{\mathrm{s}}$ are given by ${ }^{6,7}$

$$
x_{\mathrm{s}}=1 / \beta M \text {, i.e., } \sigma=1 \text { and } t_{\mathrm{s}}=\pi+1 / \beta M \text {, }
$$

for the case of $X(t)=\cos t-1$ [cf. Eq. (9)]. We shall emphasize that the shock is formed in the far field.

After the shock formation, Eq. (15) can survive with the aid of equal-areas rule: ${ }^{19}$ The shock is located in the phase so as to cut off equal areas of the multivalued wave on either side of the shock, and except for the shock, the evolution of smooth portions of the profile can still be governed by Eq. (15). The result shows that each shock grows [Figs. 3(d) and $3(\mathrm{e})]$ and in the region $\sigma \geqslant 3$ the entire wave profile can be well described by a formula ${ }^{20}$

$$
v= \begin{cases}-\frac{y}{1+\sigma} & (0 \leqslant y<\pi), \\ -\frac{y-2 \pi}{1+\sigma} & (\pi<y \leqslant 2 \pi),\end{cases}
$$

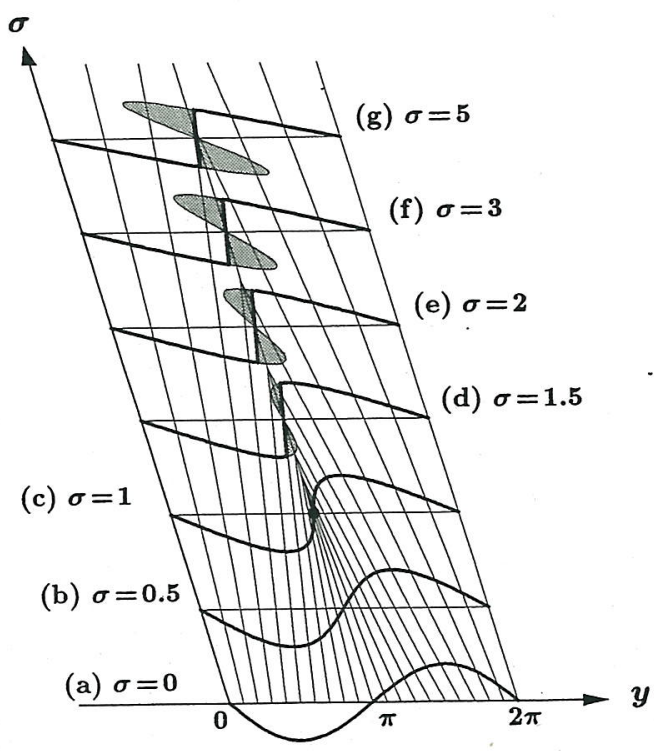

FIG. 3. Propagation process according to the conventional weakly nonlinear theory. The profiles of $v,[p-(1 / \gamma)] / M$, and $(\rho-1) / M$ are all the same. The thin solid straight lines are the characteristics. (a) The wave radiated by a harmonically oscillating plate has the sinusoidal profile at $\sigma=0$, and (b) the waveform is gradually distorted as the wave propagates. (c) At $\sigma=1$, the shock wave is formed. A small black circle denotes the shock formation point in the profile. (d)-(g) The shock discontinuity is inserted into the triple valued waveform by the equal-areas rule. The profile evolves into the sawtooth wave described by Eq. (17).

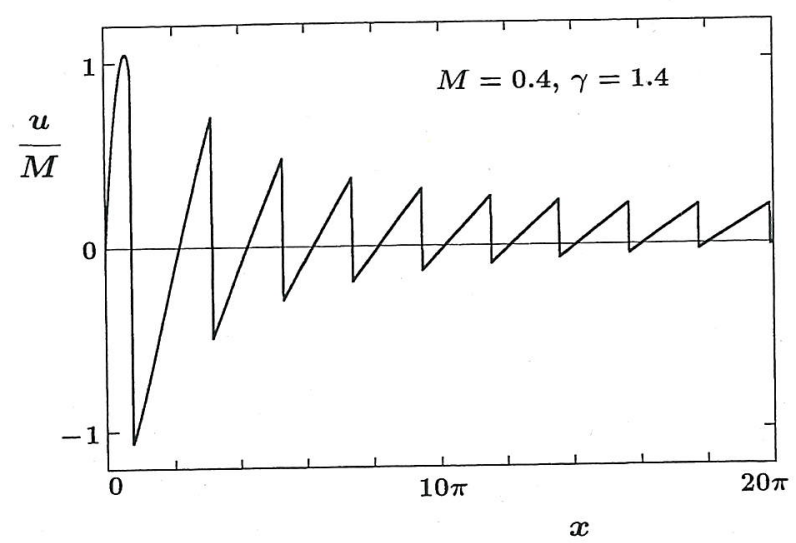

FIG. 4. Entire waveform of velocity at the time $t=20 \pi$. The head of the wave is located at $x=20 \pi$. The leading (first) shock wave swallows the negative phase (expansion wave) ahead of it.

which is periodically continued in $y$ [Figs. 3(f) and $3(\mathrm{~g})$ ]. The entire wave train is referred to as the sawtooth wave. The wave vanishes in the limit as $\sigma \rightarrow \infty$.

As shown in Fig. 3, the shock speed $V_{S}$ in the $(y, \sigma)$ plane is zero [i.e., it is $c_{0}$ in the original $\left(x^{*}, t^{*}\right)$ plane], and the waveform retains a symmetry between the rarefactive phase $(v<0)$ and the compressive phase $(v>0)$ in each wave cycle through the whole process of wave propagation. A time-averaged mass flux density is therefore zero, namely, streaming motion is not excited. Clearly, the wave phenomenon at a point $x$ is exactly repeated with period $2 \pi$ for $t>x$, i.e., the phenomenon is in a steady state.

\section{B. Strongly nonlinear wave $[M=O(1)$, Re $\gg 1]$ (Ref. 1)}

Owing to the rapid waveform distortion due to the strong nonlinearity, shock waves appear in the near field. Then the wave train is immediately transformed into an asymmetrical sawtooth-like wave (see Fig. 4), which transports the gas in the direction of wave propagation, namely, streaming is induced. The existence of quasisteady streaming is signified by the fact that the time-averaged mass flux density

$$
\overline{\rho u}(x, t)=\frac{1}{2 \pi} \int_{t-2 \pi}^{t} \rho(x, \tau) u(x, \tau) \mathrm{d} \tau,
$$

is not zero and it weakly depends on $t$ (see Fig. 5). Note that $\overline{\rho u}=0$ up to the time of shock formation. ${ }^{1}$ It has numerically been confirmed that streaming is, up to the time $t=120 \pi$, spatially almost uniform and temporally almost constant except for the top of the wave and the neighborhood of the plate. ${ }^{1}$ Such a quasisteady streaming inevitably leads to the decrease in density near the plate. In fact, the time-averaged density

$$
\bar{\rho}(x, t)=\frac{1}{2 \pi} \int_{t-2 \pi}^{t} \rho(x, \tau) \mathrm{d} \tau,
$$

decreases in the near field with increase in time (see Fig. 6). On the other hand, it has been shown that the time-averaged pressure 


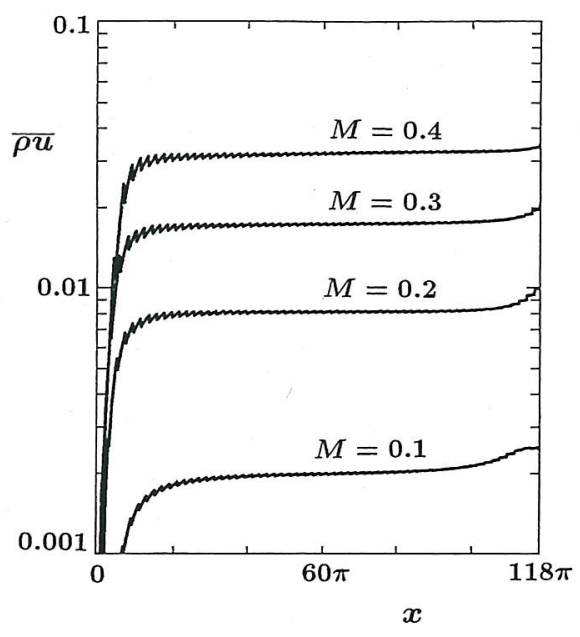

FIG. 5. The time-averaged mass flux density at $t=120 \pi$.

$$
\bar{p}(x, t)=\frac{1}{2 \pi} \int_{t-2 \pi}^{t} p(x, \tau) \mathrm{d} \tau,
$$

is hardly affected by the density reduction, ${ }^{1}$ because the entropy produced at shock fronts, which accumulates in the near field more and more as time goes by, compensates for the density reduction. Therefore, in Ref. 1, we have reached the conclusion that the phenomenon may not have the periodicity in the limit as $t \rightarrow \infty$.

Furthermore, the wave behavior in a distant region from the source has analytically been examined in Ref. 1, and thereby a simple relation between the shock speed and streaming velocity has been obtained. The result can be arranged as follows: At large distances, $\sigma=\sigma_{0}(\gg 1)$, the amplitude of the wave sufficiently decreases owing to the energy dissipation at shock fronts. The asymmetric sawtoothlike profile realized there can therefore be regarded as that composed of just the two weakly nonlinear triangular waves with compressive and rarefactive phases (see Fig. 7). The wave profile is approximately expressed by a formula

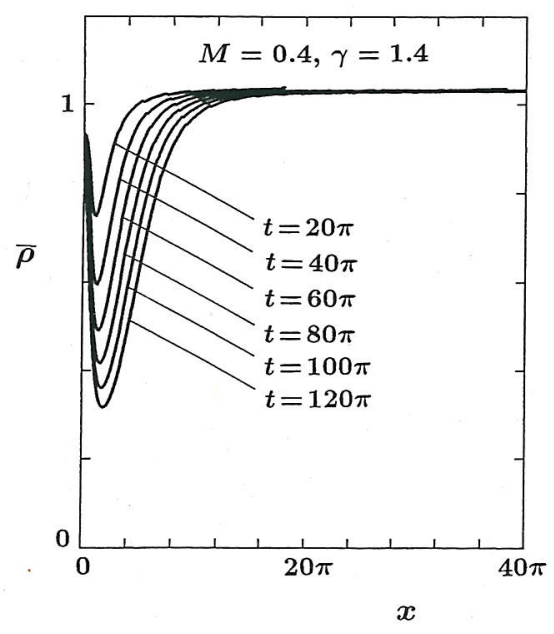

FIG. 6. The time-averaged density.

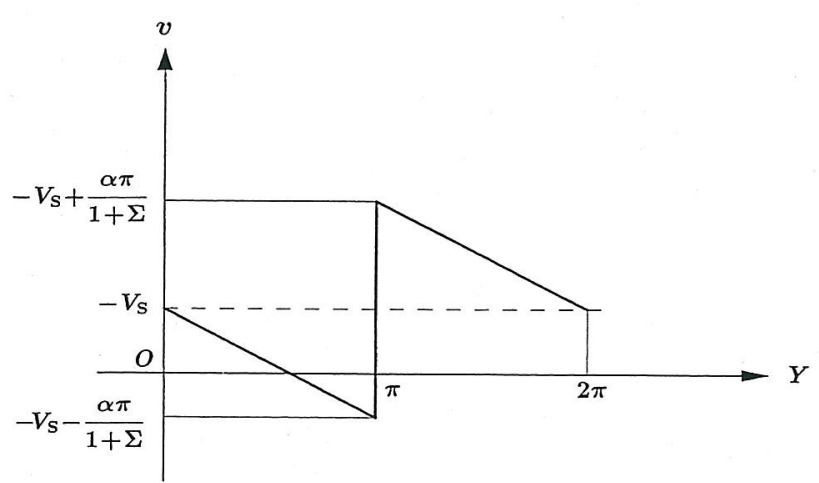

FIG. 7. Asymptotic profile of the sawtooth-like wave.

$$
v(Y, \Sigma)=\left\{\begin{array}{l}
-\frac{\alpha Y}{1+\Sigma}-V_{\mathrm{S}} \quad(0 \leqslant Y<\pi), \\
-\frac{\alpha(Y-2 \pi)}{1+\Sigma}-V_{\mathrm{S}} \quad(\pi<Y \leqslant 2 \pi) .
\end{array}\right.
$$

Here, $-\alpha$ is the slope of the triangular wave at $\sigma=\sigma_{0}$, and $\Sigma, V_{\mathrm{S}}$, and $Y$ are defined by

$$
\begin{aligned}
& \Sigma=\alpha\left(\sigma-\sigma_{0}\right), \quad V_{\mathrm{S}}=-\alpha\left(\pi-y_{\mathrm{S} 0}\right), \\
& Y=y-V_{\mathrm{S}} \sigma+\pi-\left(y_{\mathrm{S} 0}-V_{\mathrm{S}} \sigma_{0}\right),
\end{aligned}
$$

where $y_{\mathrm{SO}}(<\pi)$ is the location of the shock in the phase at $\sigma=\sigma_{0}, \quad \Sigma$ denotes the normalized distance from $\sigma_{0}$, $V_{\mathrm{S}}(<0)$ is the shock speed in the $(y, \sigma)$ plane, and $Y$ is the phase moving with the shock speed $V_{\mathrm{S}}$. We have shown ${ }^{1}$ that the shock speed $V_{\mathrm{S}}$ and a phase shift $\pi-\left(y_{\mathrm{S} 0}-V_{\mathrm{S}} \sigma_{0}\right)$ are constant independent of $\sigma_{0}$, as long as $\sigma_{0} \gg 1$ (but they depend on $M$ ). Formula (21) states that at large distances the profile approaches the superposition of the symmetric sawtooth wave with no streaming [cf. Eq. (17)] and a uniform flow $-V_{\mathrm{S}}$. By using formula (21), we readily have

$$
\overline{\rho u}=-M V_{\mathrm{S}},
$$

in the leading order of approximation. ${ }^{21}$

\section{LONG TIME BEHAVIOR OF STRONGLY NONLINEAR WAVES: NUMERICAL RESULTS}

Now we shall present the new numerical result. The calculation is extended up to the time $t=382 \pi$, which is about three times longer than the previous calculation. ${ }^{1}$ The same numerical method as in Ref. 1 is adopted, which is based on the high-resolution upwind finite difference scheme ${ }^{4}$ with Osher's flux-difference splitting. ${ }^{5}$ The scheme has been utilized by the present authors in some problems of strongly nonlinear waves, ${ }^{10,11,22}$ where the numerical method has been validated in various ways. We need not give the algorithm to construct the scheme in this paper, because it is formulated in Ref. 4 at some length (see also Ref. 5). The discretization of governing equations and numerical treatment at the oscillating boundary are explained in Refs. 1 and 10. The mesh resolutions used here were comparable to those in Ref. 1, typically, $\Delta x=2 \pi / 600$ and $\Delta t=2 \pi / 6000$. 

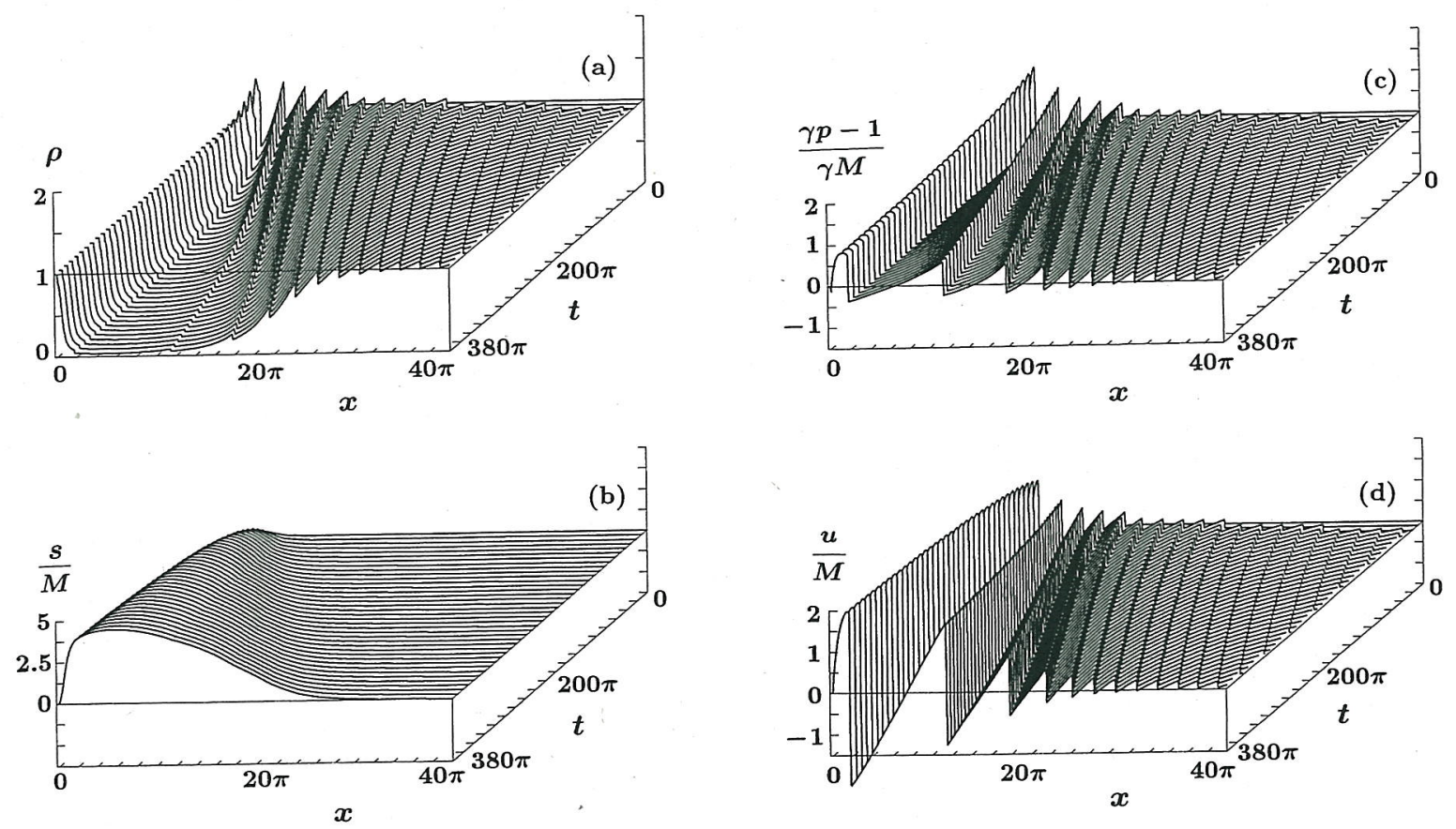

FIG. 8. The long time evolution of the wave profiles in the strongly nonlinear case of $M=0.5$ and $\gamma=1.4$. (a) Density. (b) Entropy. (c) Pressure. (d) Velocity.

The ratio of specific heats was fixed at $\gamma=1.4$. The computations were performed on the supercomputer HITAC S-820 at Hokkaido University.

\section{A. Long time evolution of the wave field}

The long time evolution of the wave field for the case of $M=0.5$ and $\gamma=1.4$ is shown in Fig. 8, where the wave profiles in a region near the plate $(0 \leqslant x \leqslant 40 \pi)$ are plotted from $t=0$ to $380 \pi$ in steps of $10 \pi$ (five periods). In Fig. 8(a) we demonstrate that the density decreases in the near field and that the linear dimension of a low-density region gradually grows as time proceeds. Clearly, the continual density reduction is caused by the quasisteady streaming. Figure 8(b) shows the distribution of entropy generated at shock fronts, where the entropy increment $s$ from the initial isentropic state is given by

$$
s=\ln \left(\gamma p / \rho^{\gamma}\right) .
$$

An appreciable entropy production occurs at shock fronts of moderate strength in the near field and a small amount of it only is carried away along the particle path by the streaming motion. Consequently, the entropy accumulates in the near field more and more as time passes. The high-entropy region nearly coincides with the low-density region. The evolution of pressure field is shown in Fig. 8(c). In contrast to the drastic change of density profile, the pressure profile at large $t$ is not considerably altered from that at small $t$, since the increase in entropy cancels the decrease in density in the near field [cf. Eq. (24)]. We exhibit the velocity field in Fig. 8(d).

As can be seen in Fig. 8, the wavelength (interval of adjacent shocks) increases in the low-density and highentropy region. Roughly speaking, by the definition of local sound speed $c^{*}=\sqrt{\gamma p^{*} / \rho^{*}}, c^{*}$ becomes very large in the region where the density is very low and the pressure is almost constant, and the time scale which characterizes the phenomenon is the period of oscillation of plate, $2 \pi / \omega$. Hence, the wavelength $2 \pi c^{*} / \omega$ increases there. The asymmetric sawtooth-like profile outside the low-density and high-entropy region is well approximated by formula (21).

\section{B. Long time behavior of streaming, time-averaged density, and pressure}

The long time evolution of streaming is shown in Fig. 9. Even at $t=382 \pi, \overline{\rho u}$ remains almost constant except for the neighborhood of the plate where $\overline{\rho u}$ nearly equals to zero. The region where $\overline{\rho u} \approx 0$ almost coincides with the region of low density and high entropy. The plateau-like portion of streaming curve in Fig. 9 shifts rightward with increase in the size of the low-density and high-entropy region. In Figs.

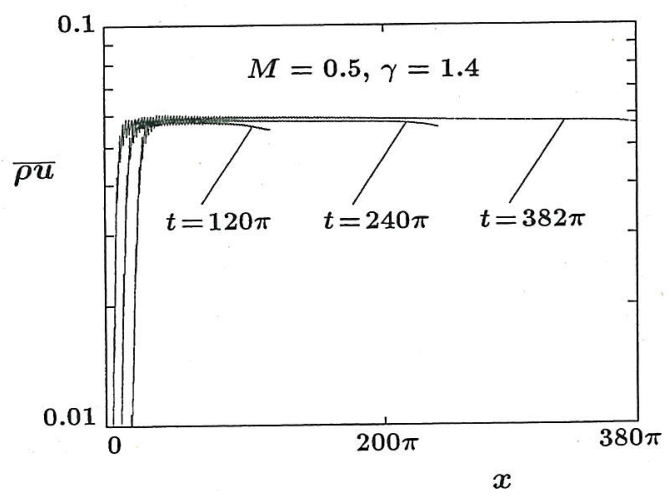

FIG. 9. The long time evolution of streaming in the case of $M=0.5$ and $\gamma=1.4$.

T. Yano and $Y$. Inoue 

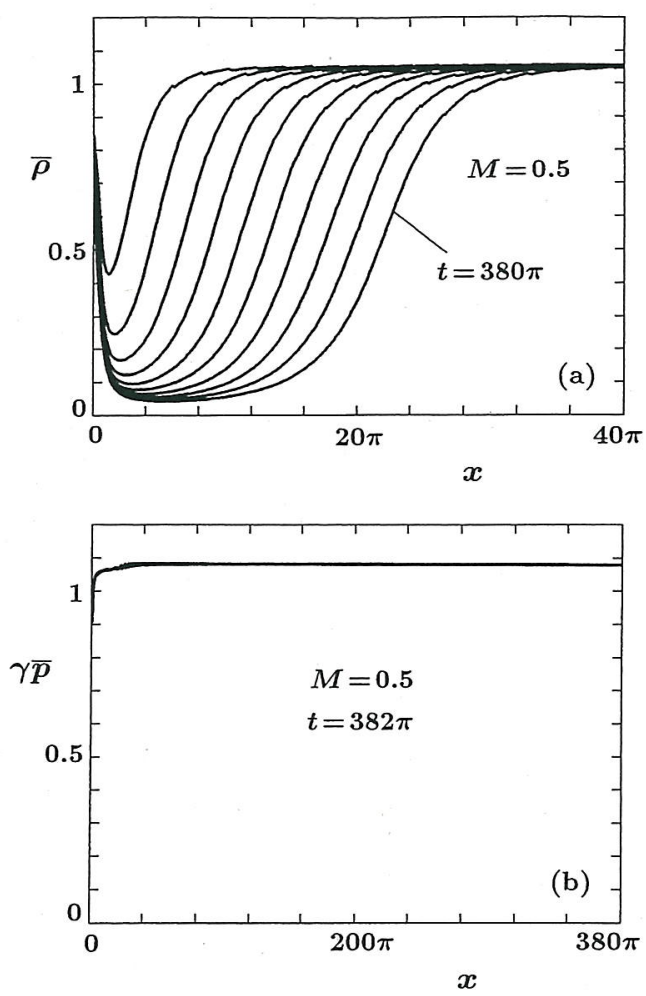

FIG. 10. The long time evolution of the time-averaged density and pressure in the case of $M=0.5$ and $\gamma=1.4$. (a) The time-averaged density. Curves are plotted from $t=38 \pi$ to $380 \pi$ in steps of $38 \pi$. (b) The time-averaged pressure at $t=382 \pi$.

10(a) and 10(b), we depict the long time behavior of $\bar{\rho}$ and $\bar{p}$, respectively. The distribution of $\bar{p}$ is not very altered [cf. Fig. 8(c)], as compared with that of $\bar{\rho}$. Figure 11 shows $\overline{\rho u}$ at $t=120 \pi$ and $x=100 \pi$ for various values of $M$. For $M \leqslant 0.4, \overline{\rho u}$ is approximately proportional to $M^{2}$.

We summarize the feature of long time evolution of wave field as follows: The nonlinear wave is emitted from the oscillating plate into a nonuniform gas, which has low density and high entropy near the plate. After the wave goes out of the region, the profile evolves into an asymmetric sawtooth-like one, which is well approximated by formula

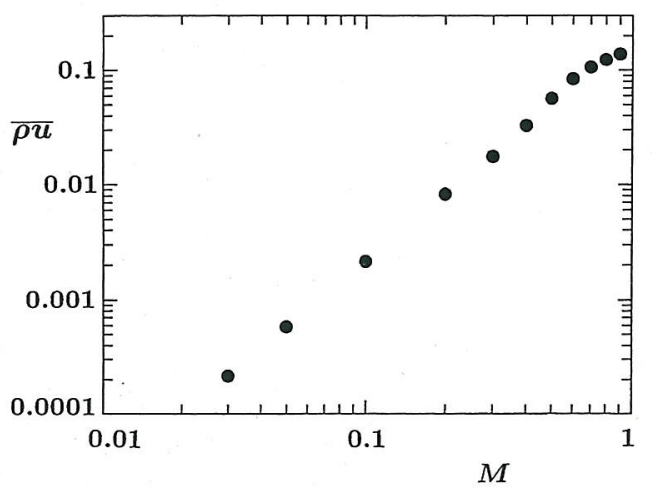

FIG. 11. Streaming for various values of $M$ at $t=120 \pi$ and $x=100 \pi$. The time-averaged mass flux density $\overline{\rho u}$ approximately varies as $0.2 M^{2}$ for $M \lesssim 0.4$.
(21). The low-density and high-entropy region continues to grow at almost constant rate. In spite of the drastic change of the field, streaming retains its quasisteadiness outside the low-density and high-entropy region, because the profile realized there can be described by formula (21), which is the superposition of the symmetric sawtooth wave and a uniform flow. Such a phenomenon may be regarded as being in a quasisteady state (see Appendix A).

\section{SECOND-ORDER ANALYSIS FOR WEAKLY NONLINEAR WAVE}

In this and the next sections, we shall verify that streaming with rarefaction effect exists even in the weakly nonlinear case and clarify the process of rarefaction and the relation between the decreasing density and the increasing entropy. To this end, we shall solve the higher-order weakly nonlinear problem of $M \ll 1$ and Re»1. Note that the results in Secs. V and VI are applicable to any function $X(t)$, as long as it is sufficiently smooth and periodic with period $2 \pi$, and $X(0)=X^{\prime}(0)=0$ [cf. Eq. (9)].

\section{A. Second-order solution including weak shocks}

Formula (15) of the conventional weakly nonlinear theory is the first-order solution. The second-order solution for the fluid velocity can be obtained as

$$
\begin{aligned}
& v=X^{\prime}(\xi)+M X(\xi) X^{\prime \prime}(\xi), \\
& \xi=y+\sigma X^{\prime}(\xi)+M \sigma X(\xi) X^{\prime \prime}(\xi)-\beta M \sigma\left[X^{\prime}(\xi)\right]^{2}
\end{aligned}
$$

(see Appendix B for the derivation and the relation of the second-order solution to the exact solution of simple wave). When the parameter $\xi$ is fixed, the second of Eq. (25) is the second-order version of the characteristic, along which the fluid velocity is constant. Substituting Eq. (25) into the condition $\partial v / \partial y=\infty$ immediately gives the distance and time of shock formation with second-order correction as $x_{\mathrm{s}}=1 / \beta M-\left[(\gamma-2)^{2} /(\gamma+1)\right] M$ and $t_{\mathrm{s}}=\pi+x_{\mathrm{s}}+2 M$. Beyond the shock formation distance $x_{s}$, the profile described by Eq. (25) becomes triple valued. As in the first-order theory reviewed in Sec. III A, since the entropy production and jump of the Riemann invariant $S$ at shock fronts can be ignored in the second-order approximation, we can use Eq. (25) to describe the continuous parts of profile after we insert a shock discontinuity into the triple valued profile and cut off the two areas in front of and behind the discontinuity. Accordingly, what we should do here is to locate the shock discontinuity to the accuracy of $O\left(M^{2}\right)$.

In the first-order theory, the equal-areas rule facilitates the determination of shock location. In the approximation of $O\left(M^{2}\right)$, we use a formula for the shock speed $U_{\mathrm{S}},{ }^{23}$

$$
\begin{aligned}
U_{\mathrm{S}}=\frac{\mathrm{d} x_{\mathrm{S}}}{\mathrm{d} t}= & 1+\beta M \frac{v_{1}+v_{2}}{2}+\beta^{2} M^{2} \frac{\left(v_{1}-v_{2}\right)^{2}}{8} \\
& +O\left(M^{3}\right),
\end{aligned}
$$

where $x_{\mathrm{S}}$ is the location of shock at the time $t$ and the subscripts 1 and 2 signify the values of immediately in front of and behind the discontinuous shock front [the equal-areas rule can be derived by using the first-order version of Eq. 
(26) and the first-order solution (15); see, e.g., Ref. 9]. We can transform Eq. (26) to give the relation connecting the shock speed on the $(y, \sigma)$ plane, $V_{\mathrm{S}}$, to $v_{1}$ and $v_{2}$,

$$
V_{\mathrm{S}}=\frac{\mathrm{d} y_{\mathrm{S}}}{\mathrm{d} \sigma}=-\frac{v_{1}+v_{2}}{2}+\beta M \frac{v_{1}^{2}+6 v_{1} v_{2}+v_{2}^{2}}{8}+O\left(M^{2}\right),
$$

where $y_{\mathrm{S}}=y_{\mathrm{S}}(\sigma)=t_{\mathrm{S}}(\sigma)-\sigma / \beta M$ is the phase of shock at a distance $\sigma=\beta M x$ and $t_{\mathrm{S}}(\sigma)$ is the time when the shock concerned reaches the point $\sigma$. Since the smooth portions of profile evolve in accordance with Eq. (25), we have

$$
v_{1}=X^{\prime}\left(\xi_{1}\right)+M X\left(\xi_{1}\right) X^{\prime \prime}\left(\xi_{1}\right)
$$

and

$$
v_{2}=X^{\prime}\left(\xi_{2}\right)+M X\left(\xi_{2}\right) X^{\prime \prime}\left(\xi_{2}\right),
$$

and $\xi_{1}$ and $\xi_{2}$ are connected to $y_{\mathrm{S}}$ via

$$
\begin{aligned}
& \xi_{1}=y_{\mathrm{S}}+\sigma X^{\prime}\left(\xi_{1}\right)+M \sigma X\left(\xi_{1}\right) X^{\prime \prime}\left(\xi_{1}\right)-\beta M \sigma\left[X^{\prime}\left(\xi_{1}\right)\right]^{2}, \\
& \xi_{2}=y_{\mathrm{S}}+\sigma X^{\prime}\left(\xi_{2}\right)+M \sigma X\left(\xi_{2}\right) X^{\prime \prime}\left(\xi_{2}\right)-\beta M \sigma\left[X^{\prime}\left(\xi_{2}\right)\right]^{2} .
\end{aligned}
$$
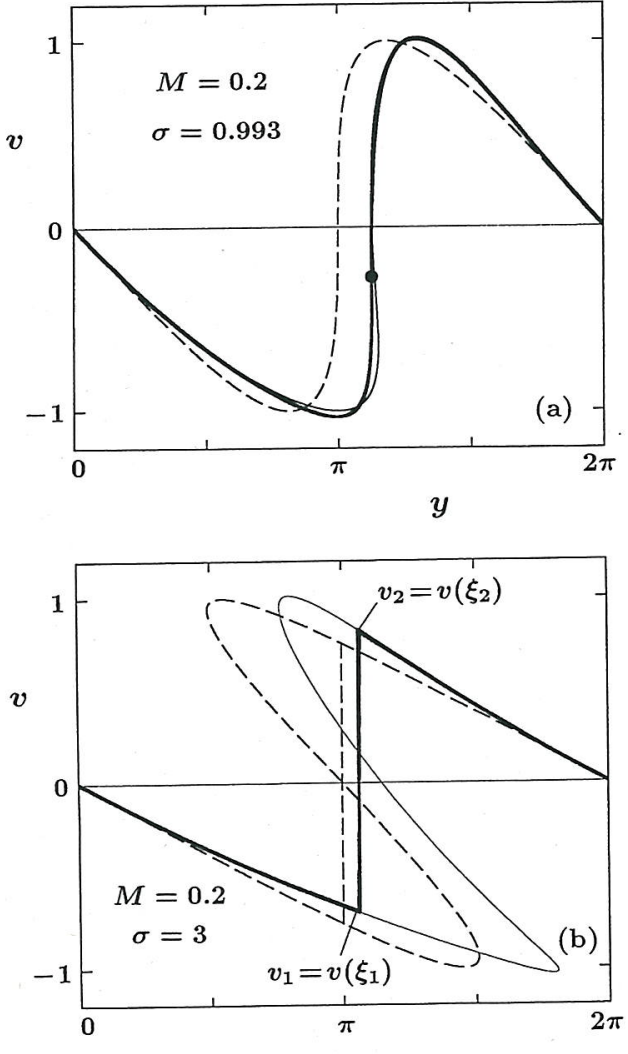

$y$

FIG. 12. The velocity profiles of the weakly nonlinear wave in the case of $M=0.2$ and $\gamma=1.4$. The bold solid line denotes the second-order solution, the dashed line the first-order solution, and the thin solid line the exact solution of simple wave, Eq. (10). (a) The profiles at the shock formation distance, $\sigma=0.993$. The black spot signifies the point of shock formation. (b) The profiles at $\sigma=3$. The numerical solution obtained by the highresolution upwind scheme completely coincides with the second-order solution.
The implicit function theorem asserts that we can find $\xi_{1}=\xi_{1}\left(y_{\mathrm{S}}, \sigma\right)$ and $\xi_{2}=\xi_{2}\left(y_{\mathrm{S}}, \sigma\right)$ as the solutions of Eqs. (29) and (30), provided that $\sigma>\beta M x_{s}$. Thus, Eqs. (27)-(30) can be regarded as an ordinary differential equation for the shock path $y=y_{\mathrm{S}}(\sigma)$. Once $y_{\mathrm{S}}(\sigma)$ is obtained, $\xi_{1}$ and $\xi_{2}$ are determined as the functions of $\sigma$.

In the case of $X(t)=\cos t-1$, the velocity profiles are shown in Fig. 12 for $M=0.2$. The difference between the second-order solution and the exact solution of simple wave is very small at the shock formation distance for such a moderately large $M$ [see Fig. 12(a)]. In Fig. 12(b), the shock discontinuity is inserted by solving Eqs. (27) and (28) with the Runge-Kutta method after inverting Eqs. (29) and (30) to yield $\xi_{1}\left(y_{\mathrm{S}}, \sigma\right)$ and $\xi_{2}\left(y_{\mathrm{S}}, \sigma\right)$ with the Newton procedure. The result for $M=0.2$ shows good agreement with the numerical solution obtained by the same high-resolution upwind scheme as used in Sec. IV.

\section{B. Weak streaming motion}

In the second-order approximation, the mass flux density $\rho u$ is

$$
\begin{aligned}
\rho u & =\left[1+u+\frac{3-\gamma}{4} u^{2}+O\left(M^{3}\right)\right] u \\
& =M v+M^{2} v^{2}+O\left(M^{3}\right),
\end{aligned}
$$

where the second of Eq. (13) has been used. This remains valid beyond the shock formation distance, because the entropy production and the jump of the Riemann invariant $S$ at the shock front are of $O\left(M^{3}\right)$. In this subsection, we shall assume that we have obtained $y_{\mathrm{S}}(\sigma), \xi_{1}=\xi_{1}(\sigma)$, and $\xi_{2}=\xi_{2}(\sigma)$ from Eqs. (27)-(30). For simplicity, we only discuss the case that just one shock is formed in each wave cycle, i.e., the number of minimum of $X^{\prime \prime}(t)$ is one in the period $2 \pi,{ }^{24}$ as in the case of $X(t)$ defined by Eq. (9).

Substituting Eq. (31) into the definition of $\overline{\rho u}$, Eq. (18), yields

$$
\begin{aligned}
\overline{\rho u}= & \frac{1}{2 \pi} \int_{0}^{\xi_{1}}\left(M v+M^{2} v^{2}\right) \frac{\partial t}{\partial \xi} \mathrm{d} \xi+\frac{1}{2 \pi} \int_{\xi_{2}}^{2 \pi}(M v \\
& \left.+M^{2} v^{2}\right) \frac{\partial t}{\partial \xi} \mathrm{d} \xi+O\left(M^{3}\right),
\end{aligned}
$$

where $\partial t / \partial \xi=\partial y / \partial \xi$ can be evaluated from the second of Eq. (25). In Eq. (32), we have shifted the range of integration to $[0,2 \pi]$ by using the periodicity of Eq. (25) for a fixed $x$. Substituting Eq. (25) into Eq. (32) and executing integration, we have 


$$
\begin{aligned}
\overline{\rho u}= & -\frac{1}{2 \pi} M\left\{X\left(\xi_{2}\right)-X\left(\xi_{1}\right)-\frac{\sigma}{2}\left(\left[X^{\prime}\left(\xi_{2}\right)\right]^{2}\right.\right. \\
& \left.\left.-\left[X^{\prime}\left(\xi_{1}\right)\right]^{2}\right)\right\}-\frac{1}{2 \pi} M^{2}\left\{X\left(\xi_{2}\right) X^{\prime}\left(\xi_{2}\right)\right. \\
& -X\left(\xi_{1}\right) X^{\prime}\left(\xi_{1}\right)+\frac{\gamma \sigma}{3}\left(\left[X^{\prime}\left(\xi_{2}\right)\right]^{3}-\left[X^{\prime}\left(\xi_{1}\right)\right]^{3}\right) \\
& \left.-\sigma\left[X\left(\xi_{2}\right) X^{\prime}\left(\xi_{2}\right) X^{\prime \prime}\left(\xi_{2}\right)-X\left(\xi_{1}\right) X^{\prime}\left(\xi_{1}\right) X^{\prime \prime}\left(\xi_{1}\right)\right]\right\} \\
& +O\left(M^{3}\right),
\end{aligned}
$$

where we have used the periodicity of $X(t)$. If a shock passes a point $\sigma$ during a period $2 \pi$ from $t-2 \pi$ to $t$, then $\xi_{1}$ and $\xi_{2}$ calculated from Eqs. (27)-(30) should be substituted into Eq. (33), and this yields nonzero $\overline{\rho u}$; otherwise one can let $\xi_{1}=\xi_{2}$ in Eq. (33) and hence $\overline{\rho u}=0$. In other words, streaming is excited in the far field beyond the shock formation distance. For $M=0.05$, Fig. 13 shows good agreement of $\bar{\rho} u$ given by Eq. (33) with the numerical results obtained in the same manner as in Sec. IV.

At sufficiently large distances, in the case of $X(t)=\cos t-1$, both $X^{\prime}\left(\xi_{1}\right)$ and $X^{\prime}\left(\xi_{2}\right)$ approach zero [cf. Eq. (25)]. This implies that $\xi_{1} \rightarrow 0$ and $\xi_{2} \rightarrow 2 \pi$ and hence $X\left(\xi_{1}\right) \rightarrow 0$ and $X\left(\xi_{2}\right) \rightarrow 0$. In the leading order of approximation, we have, from Eqs. (29) and (30), $\sigma\left[X^{\prime}\left(\xi_{2}\right)\right.$ $\left.-X^{\prime}\left(\xi_{1}\right)\right]=\xi_{2}-\xi_{1}=2 \pi$, and from Eqs. (27) and (28), $V_{\mathrm{S}}=-\left[X^{\prime}\left(\xi_{2}\right)+X^{\prime}\left(\xi_{1}\right)\right] / 2$, and consequently Eq. (33) reduces to Eq. (23).

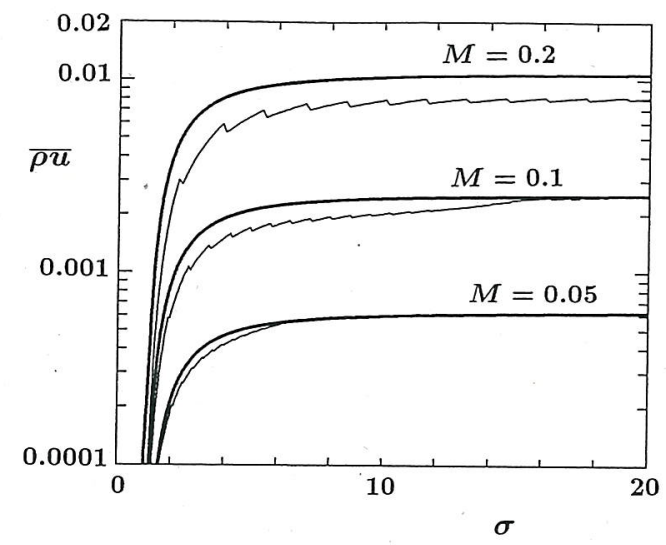

FIG. 13. Distribution of weak streaming. The bold solid lines are $\overline{\rho u} \mathrm{calcu}-$ lated by using Eq. (33) and the thin solid lines are the numerical solutions at $t=150.8$ obtained by the high-resolution upwind scheme.

Beyond the shock formation distance, streaming carries the gas by an amount of $O\left(M^{2}\right)$ per unit time and per unit area (see Fig. 13), thereby decreasing the density of the gas in a region where the streaming does not fully develop $(1<\sigma \leqslant 5)$. Since the linear dimension of the region is of $O(1 / M)$, the density reduction per unit time is of $O\left(M^{3}\right)$, and hence the determination of density distribution is not included in the present second-order analysis.

Substituting the relation $\rho=1+M v+[(3-\gamma) / 4]$ $M^{2} v^{2}+O\left(M^{3}\right)$ into Eq. (19) and using Eq. (25), we obtain the second-order version of $\bar{\rho}$ as

$$
\begin{aligned}
\bar{\rho}= & 1-\frac{1}{2 \pi} M\left\{X\left(\xi_{2}\right)-X\left(\xi_{1}\right)-\frac{\sigma}{2}\left(\left[X^{\prime}\left(\xi_{2}\right)\right]^{2}-\left[X^{\prime}\left(\xi_{1}\right)\right]^{2}\right)\right\}-\frac{1}{2 \pi} M^{2}\left\{X\left(\xi_{2}\right) X^{\prime}\left(\xi_{2}\right)-X\left(\xi_{1}\right) X^{\prime}\left(\xi_{1}\right)\right. \\
& +\frac{(5 \gamma+1) \sigma}{12}\left(\left[X^{\prime}\left(\xi_{2}\right)\right]^{3}-\left[X^{\prime}\left(\xi_{1}\right)\right]^{3}\right)-\sigma\left[X\left(\xi_{2}\right) X^{\prime}\left(\xi_{2}\right) X^{\prime \prime}\left(\xi_{2}\right)-X\left(\xi_{1}\right) X^{\prime}\left(\xi_{1}\right) X^{\prime \prime}\left(\xi_{1}\right)\right] \\
& \left.+\frac{\gamma+1}{4}\left(\int_{0}^{\xi_{1}}\left[X^{\prime}(\xi)\right]^{2} \mathrm{~d} \xi+\int_{\xi_{2}}^{2 \pi}\left[X^{\prime}(\xi)\right]^{2} \mathrm{~d} \xi\right)\right\}+O\left(M^{3}\right) .
\end{aligned}
$$

In the region up to the shock formation distance, one can let $\xi_{1}=\xi_{2}$ in Eq. (34) and hence we readily have $\bar{\rho}=1-(\beta / 4) M^{2}+O\left(M^{3}\right)$ for $X(t)=\cos t-1$; this agrees with a known result. ${ }^{25}$ Beyond the shock formation distance, however, Eq. (34) cannot account for the rarefaction effect, because the right-hand side is constant at a location $\sigma$ (see Sec. VI B).

It may here be noted that, owing to the periodicity of Eq. (25) in $t, \overline{\rho u}$ calculated from Eq. (33) is independent of $t$ at a fixed $x$; however, the mass conservation law requires such a unidirectional mean mass flow to be, at least, quasisteady. Since the $t$ dependence of $\overline{\rho u}$ may sufficiently be weak in the weakly nonlinear case, we may not need to pursue the formula for dependence of $\overline{\rho u}$ on $t$ (this will be a cumbersome task).

\section{THIRD-ORDER ANALYSIS FOR WEAKLY NONLINEAR WAVE: RAREFACTION PHENOMENON}

In this section, we shall take account of the third-order effects of production of entropy and generation of reflected wave at each shock front. It will be shown that the decrease in density of the gas can be evaluated by the careful examination of these third-order effects.

\section{A. Third-order effects at shock front}

We shall assume that $(\rho, p, u)$ satisfy Euler equations (4)-(6) everywhere except for the shock path denoted by $t=t_{\mathrm{S}}(x)^{\circ}\left(t>t_{\mathrm{S}}, x>x_{\mathrm{S}}\right)$, across which $(\rho, p, u)$ are supposed to have a jump discontinuity satisfying the RankineHugoniot relations. Then, the entropy production at a shock 
front, $\Delta s \equiv s_{2}-s_{1}$, and the jump of the Riemann invariant $S$ across the shock path, $\Delta S \equiv S_{2}-S_{1}$, are represented in the forms $^{23}$

$$
\begin{aligned}
& \Delta s=\frac{\gamma\left(\gamma^{2}-1\right)}{12}\left(u_{2}-u_{1}\right)^{3}+O\left(M^{4}\right), \\
& \Delta S=\frac{(\gamma+1)^{2}}{32}\left(u_{2}-u_{1}\right)^{3}+O\left(M^{4}\right) .
\end{aligned}
$$

At the shock formation time $t_{\mathrm{s}},(\rho, p, u)$ should match with the simple-wave solution described by Eqs. (10) and (13).

For $t>t_{\mathrm{s}}$, let $(\tilde{\rho}, \tilde{p}, \tilde{u})$ be piecewise continuous sing-levalued "solutions" obtained by cutting off the triple-valued portions of the profiles described by Eqs. (10) and (13) at shock fronts. The continuous parts of $(\tilde{\rho}, \tilde{p}, \widetilde{u})$ satisfy Euler equations (4)-(6), but the Rankine-Hugoniot relations are not satisfied at discontinuities. Since $\Delta s$ and $\Delta S$ are of $O\left(M^{3}\right)$, the deviation of $(\rho, p, u)$ from $(\widetilde{\rho}, \widetilde{p}, \widetilde{u})$ may be regarded as being of $O\left(M^{3}\right)$; we shall express the deviation as $(\hat{\rho}, \hat{p}, \hat{u})$. Thus, we decompose $(\rho, p, u)$ into $(\widetilde{\rho}, \widetilde{p}, \widetilde{u})$ and the correction terms $(\hat{\rho}, \hat{p}, \hat{u})$ as follows:

$$
\rho=\tilde{\rho}+\hat{\rho}, \quad p=\tilde{p}+\hat{p}, u=\tilde{u}+\hat{u},
$$

where $\tilde{\rho}-1=O(M), \quad \tilde{p}-1 / \gamma=O(M), \quad \tilde{u}=O(M)$, and $(\hat{\rho}, \hat{p}, \hat{u})$ are of $O\left(M^{3}\right)$. Making use of the jump conditions (35) and Eq. (36), we shall derive the formula which determines the amplitude of a reflected wave at shock fronts, and then we shall seek the correction terms explicitly up to $O\left(M^{3}\right)$.

Substituting Eq. (36) into Eq. (35) gives

$$
\begin{aligned}
& \hat{p}_{2}-\hat{\rho}_{2}-\hat{p}_{1}+\hat{\rho}_{1}=\frac{\gamma^{2}-1}{12}\left(\widetilde{u_{2}}-\widetilde{u}_{1}\right)^{3}+O\left(M^{4}\right), \\
& \frac{\gamma \hat{p}_{2}-\hat{\rho}_{2}}{\gamma-1}-\hat{u}_{2}-\frac{\gamma \hat{p}_{1}-\hat{\rho}_{1}}{\gamma-1}+\hat{u}_{1} \\
& =\frac{(\gamma+1)^{2}}{32}\left(\widetilde{u_{2}}-\widetilde{u_{1}}\right)^{3}+O\left(M^{4}\right),
\end{aligned}
$$

where we have used the fact that $\tilde{s}=\ln \left(\gamma \tilde{p} / \tilde{\rho}^{\gamma}\right)=0$ and $\widetilde{S}=\widetilde{u}-[2 /(\gamma-1)] \sqrt{\gamma \widetilde{p} / \widetilde{\rho}}=-2 /(\gamma-1)$ everywhere. On the other hand, substituting Eq. (36) into Euler equations (4)-(6) and neglecting terms of $O\left(M^{4}\right)$ yields the governing equations for $(\hat{\rho}, \hat{p}, \hat{u})$

$$
\frac{\partial \hat{p}}{\partial t}+\frac{\partial \hat{u}}{\partial x}=0, \frac{\partial \hat{u}}{\partial t}+\frac{\partial \hat{p}}{\partial x}=0, \frac{\partial \hat{p}}{\partial t}-\frac{\partial \hat{\rho}}{\partial t}=0
$$

Here, we have used the fact that $(\tilde{\rho}, \tilde{p}, \tilde{u})$ satisfies Euler equations everywhere except for the discontinuities, i.e., the shock paths. The linear system (38) is also valid everywhere except for shock paths, across which $(\hat{\rho}, \hat{p}, \hat{u})$ are discontinuous.

The general solution to the system (38) can readily be obtained as

$$
\left(\begin{array}{l}
\hat{\rho} \\
\hat{p} \\
\hat{u}
\end{array}\right)=\left(\begin{array}{c}
-1 \\
-1 \\
1
\end{array}\right) f(t+x)+\left(\begin{array}{l}
1 \\
1 \\
1
\end{array}\right) g(t-x)+\left(\begin{array}{c}
-1 \\
0 \\
0
\end{array}\right) h(x),
$$

where $f, g$, and $h$ are arbitrary functions, the functional form of which are determined by imposing the boundary conditions, i.e., the jump conditions across the shock, Eq. (37), or the boundary condition on the plate. In a region where $(\hat{\rho}, \hat{p}, \hat{u})$ are continuous, $f(t+x)$ is a left-running component constant along the linear leftward characteristic $t+x=$ const. and $g(t-x)$ a right-running component constant along the linear rightward characteristic $t-x=$ const. Entropy has a nonuniform stationary distribution $s=\gamma(\hat{p}-\hat{\rho})=\gamma h(x)$ within the approximation concerned. The jump conditions (37) can now be rewritten into

$$
f_{2}-f_{1}=A\left(\widetilde{u_{2}}-\widetilde{u_{1}}\right)^{3}, h_{2}-h_{1}=B\left(\widetilde{u_{2}}-\widetilde{u_{1}}\right)^{3},
$$

where

$$
A=\frac{(5-3 \gamma)(\gamma+1)}{192}, B=\frac{\gamma^{2}-1}{12}
$$

The first of Eq. (40) gives the amplitude of the reflected wave generated at a shock front and the second is the wellknown formula for the production of entropy ${ }^{23}$ [the latter is essentially equivalent to the first of Eq. (35)]. From Eq. (41), we have $A=0.01$ and $B=0.08$ for $\gamma=1.4$. That is, for air, the amount of entropy produced at a shock front is eight times as large as the amplitude of the reflected wave.

Formula for the amplitude of a reflected wave has been obtained by Lighthill ${ }^{12}$ and by Morfey and Sparrow ${ }^{14}$ (see also Ref. 26). The first of Eq. (40) is equivalent to the results in Refs. 12 and 14 and the derivation demonstrated above is considerably simple compared with the methods employed in the earlier works.

We shall remark that the jump conditions do not restrict the functional form of the right-running component $g(t-x)$. The function $g$ will be determined by using the boundary condition on the plate (see below).

\section{B. Accumulation of entropy and propagation of reflected wave}

By $t_{\mathrm{S}}^{(n)}(x)$ ( $n$ is a positive integer), we signify the time when the $n$th shock (enumerated from the top of the wave) reaches a point $x\left(>x_{\mathrm{S}}\right)$. The curve $t=t_{\mathrm{S}}^{(n)}(x)$ denotes the path of the $n$th shock in the $(x, t)$ plane. In Fig. 14, we schematically illustrate the shock paths in the case of $X(t)$ defined by Eq. (9) together with the linear leftward and rightward characteristics.

The region between the paths of $n$th shock and $(n+1)$ th shock in the $(x, t)$ plane can be expressed as

$$
\begin{aligned}
C^{(n)}= & \left\{(x, t) \mid t_{\mathrm{S}}^{(n)}(x)<t<t_{\mathrm{S}}^{(n+1)}(x), x_{\mathrm{s}}<x\right\} \\
& (n=1,2, \ldots)
\end{aligned}
$$

(see Fig. 14). We here note that the shock formation distance $x_{\mathrm{s}}$ is constant for a given $M$ irrespective of $n$ in the approximation considered here. The correction terms $(\hat{\rho}, \hat{p}, \hat{u})$ are continuous in the region $C^{(n)}$, but discontinuous across the shock paths. Applying the jump conditions (40) on the path of $n$th shock gives 


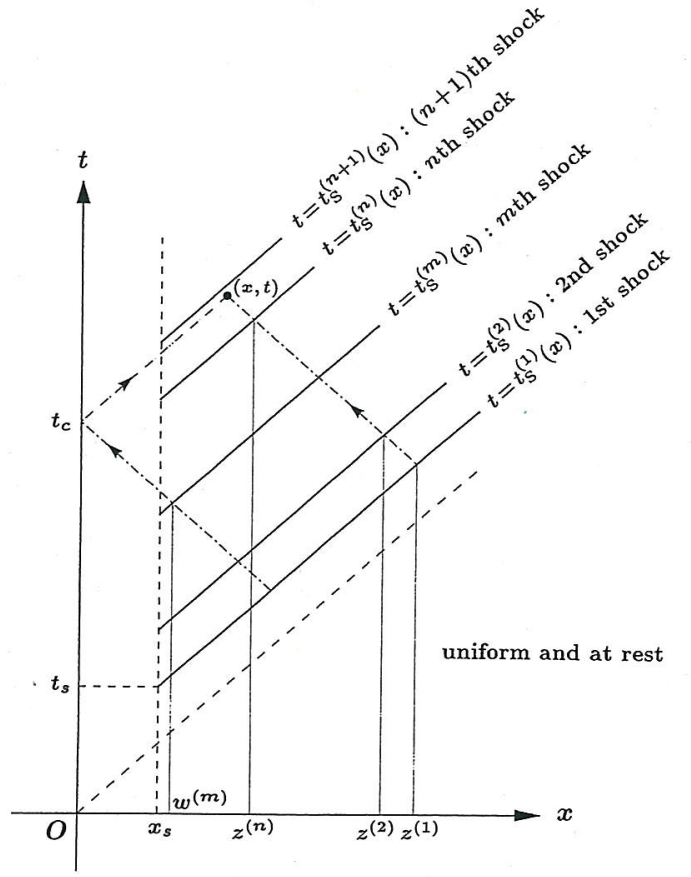

FIG. 14. Schematic of the paths of shocks in the $(x, t)$ plane. The bold solid lines are the shock paths, the dash-dotted lines are the linear leftward and rightward characteristics. A leftward characteristic $t+x=t_{\mathrm{S}}^{(m)}\left(w^{(m)}\right)+w^{(m)}$ intersects the $t$ axis at $t=t_{c}=t_{S}^{(m)}\left(w^{(m)}\right)+w^{(m)}$.

$$
f_{2}^{(n)}=f_{1}^{(n)}+A\left[\tilde{u}_{2}^{(n)}-\tilde{u}_{1}^{(n)}\right]^{3}
$$

and

$$
h_{2}^{(n)}=h_{1}^{(n)}+B\left[\tilde{u}_{2}^{(n)}-\widetilde{u}_{1}^{(n)}\right]^{3},
$$

where the superscript ${ }^{(n)}$ represents the value on the path of $n$th shock. We shall remark that $f_{1}^{(1)}=h_{1}^{(1)}=0$ because the region ahead of the first shock is the simple-wave region. From the second of Eq. (43) and the fact that $h_{1}^{(i+1)}(x)$ $=h_{2}^{(i)}(x)(i=1,2, \ldots, n-1)$, we can immediately obtain

$$
\begin{aligned}
h(x)=h_{2}^{(n)}(x)= & B \sum_{i=1}^{n}\left[\tilde{u}_{2}^{(i)}\left(x, t_{\mathrm{S}}^{(i)}(x)\right)\right. \\
& \left.-\tilde{u}_{1}^{(i)}\left(x, t_{\mathrm{S}}^{(i)}(x)\right)\right]^{3},
\end{aligned}
$$

in the region $C^{(n)}$. For $x<x_{\mathrm{s}}$, clearly, $h \equiv 0$.

Suppose that a linear leftward characteristic, $t+x=$ const. passes a given point $(x, t)$ in the region $C^{(n)}$. Then, the intersection point of the characteristic and the path of $k$ th shock, $\left(z^{(k)}, t_{\mathrm{S}}^{(k)}\left(z^{(k)}\right)\right)$, can be given by solving

$$
t+x=t_{\mathrm{S}}^{(k)}\left(z^{(k)}\right)+z^{(k)} \quad(k=1,2, \ldots, n),
$$

where $z^{(k)}$ is the $x$ coordinate of the intersection point of the path of $k$ th shock and the characteristic (see Fig. 14). Along the characteristic, a relation

$$
\begin{aligned}
f_{1}^{(i+1)}\left(t_{\mathrm{S}}^{(i+1)}\left(z^{(i+1)}\right)+z^{(i+1)}\right)= & f_{2}^{(i)}\left(t_{\mathrm{S}}^{(i)}\left(z^{(i)}\right)+z^{(i)}\right) \\
& (i=1,2, \ldots, n-1),
\end{aligned}
$$

holds. Accordingly, at a point $(x, t)$ in the region $C^{(n)}$, the left-running component $f$ is given by

$$
\begin{aligned}
f(t+x)= & f_{2}^{(n)}\left(t_{\mathrm{S}}^{(n)}\left(z^{(n)}\right)+z^{(n)}\right) \\
= & A \sum_{i=1}^{n}\left[\tilde{u}_{2}^{(i)}\left(z^{(i)}, t_{\mathrm{S}}^{(i)}\left(z^{(i)}\right)\right)\right. \\
& \left.-\widetilde{u}_{1}^{(i)}\left(z^{(i)}, t_{\mathrm{S}}^{(i)}\left(z^{(i)}\right)\right)\right]^{3}
\end{aligned}
$$

and $f(t+x)$ is continuously extended to a region $x<x_{s}$ along the characteristic (45).

The functional form of the right-running component $g(t-x)$ can be determined as follows: Suppose that a reflected wave $f(t+x)$ propagates along the leftward characteristic $t+x=t_{c} \equiv t_{S}^{(m)}\left(w^{(m)}\right)+w^{(m)}$ and it reaches $x=0$ at the time $t_{i}=t_{c}$, where $m$ is the number of shock paths intersected by the characteristic, and $w^{(m)}$ is the $x$ coordinate of the intersection point of path of $m$ th shock and the characteristic (see Fig. 14). Since the boundary condition (8) has already been satisfied by $(\widetilde{\rho}, \widetilde{p}, \widetilde{u})$, i.e., the simple-wave solution (10) and (13), the boundary condition for $\hat{u}$ on the plate is reduced to

$$
\hat{u}=0, \quad \text { i.e., } g(t)=-f(t) \text { at } x=0,
$$

within the approximation concerned. The reflected wave is reflected at $x=0$, and thereafter propagates as a rightrunning wave $g(t-x)$, which is constant along the characteristic

$$
t-x=t_{c}=t_{\mathrm{S}}^{(m)}\left(w^{(m)}\right)+w^{(m)}
$$

(see Fig. 14). By using Eq. (49) and the boundary condition (48), we can determine the functional form of $g$ as

$$
\begin{aligned}
g(t-x)= & -f_{2}^{(m)}\left(t_{\mathrm{S}}^{(m)}\left(w^{(m)}\right)+w^{(m)}\right) \\
= & -A \sum_{i=1}^{m}\left[\tilde{u}_{2}^{(i)}\left(w^{(i)}, t_{\mathrm{S}}^{(i)}\left(w^{(i)}\right)\right)\right. \\
& \left.-\widetilde{u}_{1}^{(i)}\left(w^{(i)}, t_{\mathrm{S}}^{(i)}\left(w^{(i)}\right)\right)\right]^{3} .
\end{aligned}
$$

Consequently, we have

$$
\begin{aligned}
\hat{\rho}(x, t)= & -A \sum_{i=1}^{n}\left[\tilde{u}_{2}^{(i)}\left(z^{(i)}, t_{\mathrm{S}}^{(i)}\left(z^{(i)}\right)\right)\right. \\
& \left.-\tilde{u}_{1}^{(i)}\left(z^{(i)}, t_{\mathrm{S}}^{(i)}\left(z^{(i)}\right)\right)\right]^{3} \\
& -A \sum_{i=1}^{m}\left[\tilde{u}_{2}^{(i)}\left(w^{(i)}, t_{\mathrm{S}}^{(i)}\left(w^{(i)}\right)\right)\right. \\
& \left.-\widetilde{u}_{1}^{(i)}\left(w^{(i)}, t_{\mathrm{S}}^{(i)}\left(w^{(i)}\right)\right)\right]^{3} \\
& -B \sum_{i=1}^{n}\left[\tilde{u}_{2}^{(i)}\left(x, t_{\mathrm{S}}^{(i)}(x)\right)-\tilde{u}_{1}^{(i)}\left(x, t_{\mathrm{S}}^{(i)}(x)\right)\right]^{3},
\end{aligned}
$$

$$
\begin{aligned}
\hat{p}(x, t)= & -A \sum_{i=1}^{n}\left[\tilde{u}_{2}^{(i)}\left(z^{(i)}, t_{\mathrm{S}}^{(i)}\left(z^{(i)}\right)\right)\right. \\
& \left.-\tilde{u}_{1}^{(i)}\left(z^{(i)}, t_{\mathrm{S}}^{(i)}\left(z^{(i)}\right)\right)\right]^{3} \\
& -A \sum_{i=1}^{m}\left[\tilde{u}_{2}^{(i)}\left(w^{(i)}, t_{\mathrm{S}}^{(i)}\left(w^{(i)}\right)\right)\right. \\
& \left.-\widetilde{u}_{1}^{(i)}\left(w^{(i)}, t_{\mathrm{S}}^{(i)}\left(w^{(i)}\right)\right)\right]^{3},
\end{aligned}
$$



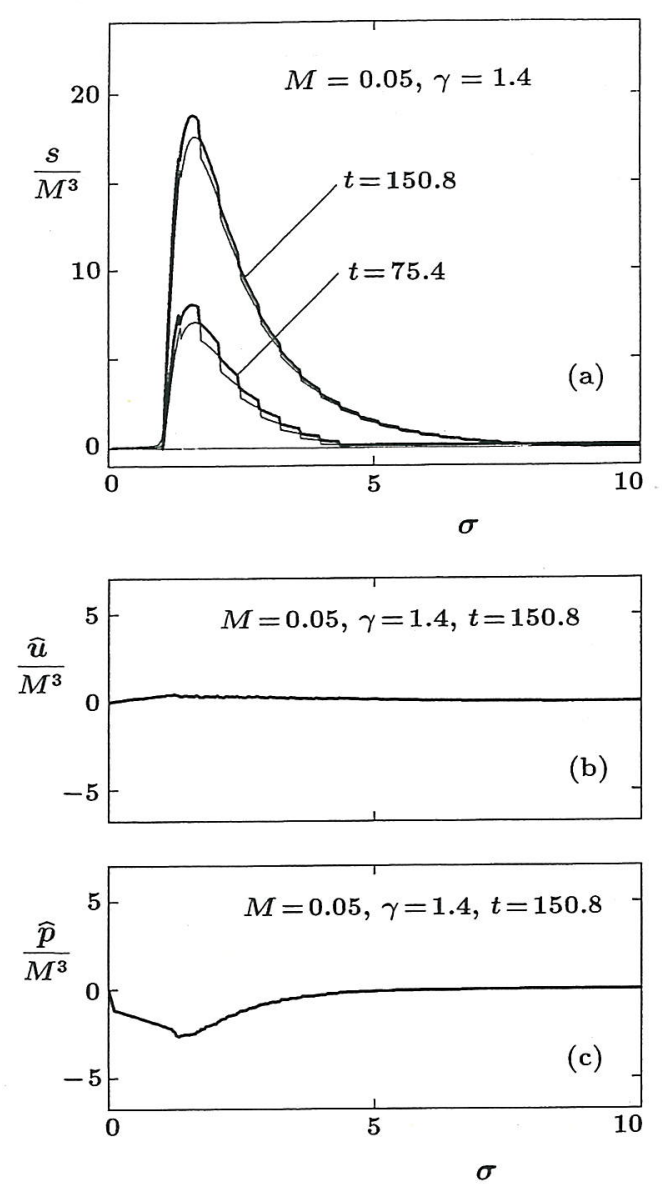

FIG. 15. (a) Distribution of entropy produced at shock fronts. The bold solid lines are $s / M^{3}=\gamma(\hat{p}-\hat{\rho})$, and the thin solid lines denote numerical results. (b) $\hat{u}$ for $M=0.05$ at $t=150.8$. (c) $\hat{p}$ for $M=0.05$ at $t=150.8$.

$$
\begin{aligned}
\hat{u}(x, t)= & A \sum_{i=1}^{n}\left[\tilde{u}_{2}^{(i)}\left(z^{(i)}, t_{\mathrm{S}}^{(i)}\left(z^{(i)}\right)\right)\right. \\
& \left.-\tilde{u}_{1}^{(i)}\left(z^{(i)}, t_{\mathrm{S}}^{(i)}\left(z^{(i)}\right)\right)\right]^{3} \\
& -A \sum_{i=1}^{m}\left[\tilde{u}_{2}^{(i)}\left(w^{(i)}, t_{\mathrm{S}}^{(i)}\left(w^{(i)}\right)\right)\right. \\
& \left.-\widetilde{u}_{1}^{(i)}\left(w^{(i)}, t_{\mathrm{S}}^{(i)}\left(w^{(i)}\right)\right)\right]^{3},
\end{aligned}
$$

where $z^{(i)}$ and $w^{(i)}$ are determined by Eqs. (45) and (49), respectively, and $n$ and $m$ are the numbers of shock paths which are intersected by the leftward characteristics. We shall remark that $\hat{\rho}$ and $\hat{p}$ are negative for $1<\gamma<5 / 3$ because $A$ and $B$ are positive and $\widetilde{u_{2}}>\widetilde{u_{1}}$.

In order to calculate the correction terms $(\hat{\rho}, \hat{p}, \hat{u})$, it is necessary to give the shock path $t=t_{\mathrm{S}}^{(n)}$ and the velocities on it, i.e., $\widetilde{u}_{2}^{(n)}$ and $\widetilde{u}_{1}^{(n)}$. Since we are concerned with the third-order corrections, without loss of accuracy, we can utilize the first-order solution for them in the following way: Applying the equal-areas rule to Eq. (15) in the case of $X(t)=\cos t-1$, we have, for $n$th shock $(n=1,2, \ldots)$,

$$
\begin{aligned}
y_{\mathrm{S}}^{(n)}(x)=t_{\mathrm{S}}^{(n)}(x)-x= & (2 n-1) \pi, \\
& \text { i.e., } t_{\mathrm{S}}^{(n)}(x)=(2 n-1) \pi+x
\end{aligned}
$$

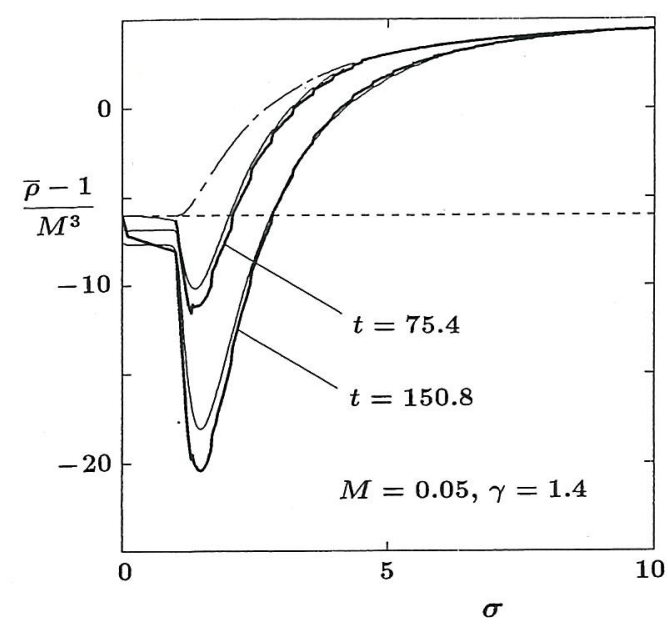

FIG. 16. Distribution of the time-averaged density. The bold solid lines are the results with the third-order corrections and the thin solid lines are numerical ones. The result up to the second order, Eq. (34), is shown by the dash-dotted line and the averaged density before the shock formation time, $\bar{\rho}=1-[(\gamma+1) / 8] M^{2}$, is denoted by the broken line.

(see Fig. 3), and from the second of Eq. (15),

$$
\begin{aligned}
\arcsin v_{1}^{(n)}+\beta M x v_{1}^{(n)}= & y_{S}^{(n)}(x) \\
= & \arcsin v_{2}^{(n)}+\beta M x v_{2}^{(n)} \\
& \left(v_{1}^{(n)}<0<v_{2}^{(n)}\right),
\end{aligned}
$$

for $\beta M x>1$ [cf. Eq. (16)]. We can solve Eq. (55) numerically to yield $v_{1}^{(n)}=v_{1}^{(n)}(x)$ and $v_{2}^{(n)}=v_{2}^{(n)}(x)$, and thus we can determine $\tilde{u}_{1}^{(n)}$ and $\widetilde{u}_{2}^{(n)}$ as

$$
\tilde{u}_{1}^{(n)}=M v_{1}^{(n)} \text { and } \tilde{u}_{2}^{(n)}=M v_{2}^{(n)} \text {. }
$$

Substituting Eqs. (54) and (56) into Eqs. (51)-(53), we can evaluate the correction terms in the approximation of $O\left(M^{3}\right)$.

Figure 15(a) shows the distribution of entropy $s=\gamma(\hat{p}-\hat{\rho})$ for $M=0.05$ and $\gamma=1.4$ in the case of $X(t)=\cos t-1$. The results agree with numerical ones by the high-resolution upwind scheme. We also show $\hat{u}$ and $\hat{p}$ in Figs. 15(b) and 15(c), which are not affected by the entropy production [see Eqs. (52) and (53)], and hence they are not so large as compared with the entropy plotted in Fig. 15(a).

In Fig. 16, we present the distribution of time-averaged density. In the weakly nonlinear case, the velocity jump $\widetilde{u_{2}}-\widetilde{u_{1}}$ at the shock fronts varies slowly as the shock propagates, and therefore $\hat{\rho}$ hardly changes during a period $2 \pi$ from $t-2 \pi$ to $t$ at a point $x$. The time-averaged density can therefore be given as the sum of $\hat{\rho}$ and the time average of $\widetilde{\rho}$, without loss of accuracy. However, it is difficult to calculate the time average of $\tilde{\rho}$ to the accuracy of $O\left(M^{3}\right)$, because, to do so, we should determine the shock path in the same accuracy. In Fig. 16, we plot the sum of $\hat{\rho}$ and Eq. (34), i.e., the second-order version of $\bar{\rho}$. This procedure involves the error of $O\left(M^{3}\right)$, and hence, strictly speaking, the result is invalid. Nevertheless, the result shows good agreement with the numerical one, as shown in Fig. 16. This is the reflection of the fact that the rarefaction effect is connected to the accumulative property of $\hat{\rho}$, while the error introduced by re- 
placing the time average of $\widetilde{\rho}$ with Eq. (34) remains small. Since $B$ is eight times larger than $A$ when $\gamma=1.4$, the main contribution to the accumulative $\hat{\rho}$ is the production of entropy at shock fronts.

\section{CONCLUSIONS AND COMMENTS}

We have carried out the large-scale computations for the strongly nonlinear problem, thereby demonstrating that the quasisteady state is established after a substantial amount of time has lapsed, where the low-density and high-entropy region formed near the plate continues to grow at almost constant rate and outside the region the quasisteady streaming endures.

The higher-order weakly nonlinear analysis presented in Secs. V and VI has given not only qualitatively but also quantitatively accurate results, as compared with the exact solution of simple wave and the numerical results by the high-resolution upwind finite difference scheme [see Figs. 12, 15(a) and 16]. As demonstrated in Figs. 15(a) and 16, the entropy accumulates as time goes by, and this is directly related to the gradual decrease in density of the gas.

In the course of time, the entropy and $\hat{\rho}$ increase and, ultimately, may become of $O\left(M^{2}\right)$, and at that time the present analysis ceases to be valid. However, as confirmed by the numerical analysis in Sec. IV, even after the density and entropy fields are changed drastically, the quasisteadiness of the phenomenon is not altered. Accordingly, the mechanism of rarefaction clarified in Sec. VI may, at least qualitatively, be correct even after the present analysis ceases to be valid.

Finally, we shall remark that we have confirmed that the mass conservation law

$$
\int_{0}^{\infty}(\rho-1) \mathrm{d} x=0
$$

is satisfied within an allowable error, when $M v+[(3-\gamma) / 4] M^{2} v^{2}+\hat{\rho}$ is substituted into $\rho-1$ in Eq. (57), where $v$ is evaluated with using Eqs. (25) and (27)(30).

\section{APPENDIX A: TIME-AVERAGED ENERGY AND MOMENTUM FLUXES}

In the following, we shall demonstrate that both the mean energy flow and mean momentum flow are quasisteady, as well as the mean mass flow $\overline{\rho u}$.

The time-averaged energy flux $\mathscr{E}$ and time-averaged momentum flux $\mathscr{C} b$ are defined as

$$
\mathscr{E}(x, t)=\frac{1}{2 \pi} \int_{t-2 \pi}^{t}\left(E_{\mathrm{t}}+p\right) u \mathrm{~d} t,
$$

and

$$
\mathscr{H}(x, t)=\frac{1}{2 \pi} \int_{t-2 \pi}^{t}\left(\rho u^{2}+p-\frac{1}{\gamma}\right) \mathrm{d} t,
$$

which, respectively, signify the time average of the total energy and momentum transported through the point $x$ by the wave motion. The distribution of $\mathscr{E}$ is plotted in Fig. 17 and

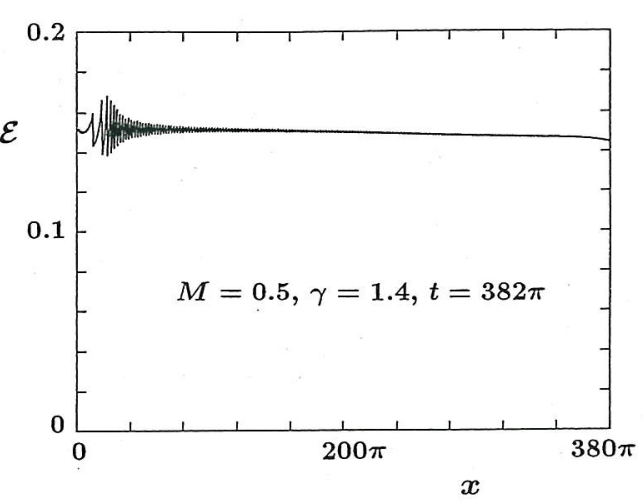

FIG. 17. Distribution of the time-averaged energy flux.

that of $\mathscr{C}$ in Fig. 18, which are computed by using the high-resolution upwind scheme. Both $\mathscr{E}$ and $\mathscr{O} b$ are almost uniform everywhere.

Before the shock formation time $t_{\mathrm{s}}$, the exact solution of the simple wave is precisely periodical with period $2 \pi$ at a fixed point $x$. By making use of the simple-wave solution, we can analytically calculate $\mathscr{E}$ and $\mathscr{b}$ for $t<t_{\mathrm{s}}$ (Ref. 22)

$$
\begin{aligned}
\mathscr{E} & =\frac{1}{2 \pi} \int_{0}^{2 \pi} p(M X(t), t) u(M X(t), t) \mathrm{d} t \\
& =\frac{1}{2} M^{2} F\left(\frac{-1}{\gamma-1}, \frac{-(\gamma+1)}{2(\gamma-1)}, 2 ; \frac{(\gamma-1)^{2}}{4} M^{2}\right) \equiv \mathscr{E}_{0},
\end{aligned}
$$

and

$$
\begin{aligned}
\mathscr{M}_{b} & =\frac{1}{2 \pi} \int_{0}^{2 \pi[}\left[p(M X(t), t)-\frac{1}{\gamma}\right] \mathrm{d} t \\
& =\frac{1}{\gamma} F\left(\frac{-\gamma}{\gamma-1}, \frac{-(\gamma+1)}{2(\gamma-1)}, 1 ; \frac{(\gamma-1)^{2}}{4} M^{2}\right)-\frac{1}{\gamma} \\
& \equiv \mathscr{M}_{0},
\end{aligned}
$$

where $M X(t)$ is the instantaneous location of oscillating plate [see Eqs. (8) and (9)] and $F(a, b, c ; z)$ is the hypergeometric function. Clearly, $\mathscr{E}_{0}$ and $\mathscr{C}_{0}$ are independent of not only $t$ but also $x$ for $t<t_{\mathrm{s}}$ and $x<t-2 \pi$. In the case of $\gamma=1.4, \mathscr{E}_{0}$ and $\mathscr{C}_{0}$ are reduced to polynomials, ${ }^{22}$

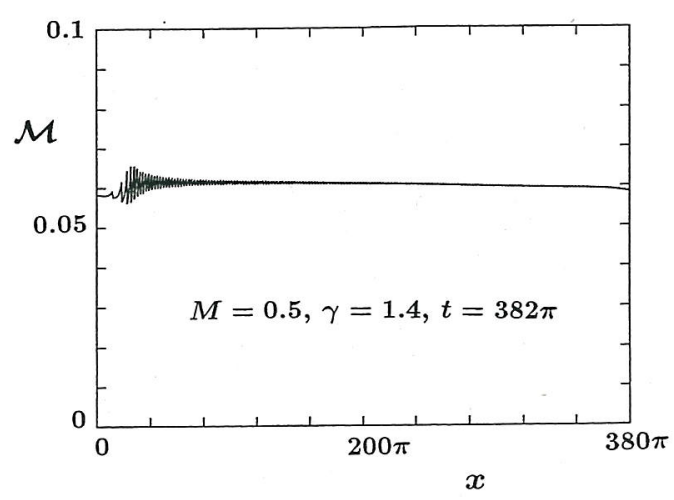

FIG. 18. Distribution of the time-averaged momentum flux. 
$\mathscr{E}_{0}=\frac{1}{2} M^{2}+\frac{3}{40} M^{4}+\frac{3}{2000} M^{6}+\frac{1}{4 \cdot 10^{5}} M^{8}$

and

$$
\mathscr{C}_{0}=\frac{3}{10} M^{2}+\frac{3}{200} M^{4}+\frac{1}{10000} M^{6} .
$$

From Eqs. (A5) and (A6), we have $\mathscr{E}_{0}=0.13$ and $\mathscr{C}_{0}=0.076$ for $M=0.5$. Strictly speaking, Eqs. (A3) and (A4), and hence (A5) and (A6), are limited to the case with a moderately small $M$, where $t_{\mathrm{s}}>2 \pi(M<0.249) .{ }^{22}$ However, since the influence of the shock is negligible at $x=0$ for $0<t<2 \pi$ when $M=0.5$, we may use $\mathscr{E}_{0}$ and $\mathscr{C}_{0}$ as the values of $\mathscr{E}$ and $\mathscr{C}$ at $x=0$ and $t=2 \pi$. As shown in Figs. 17 and 18 , the change of the distribution of the energy flux and that of momentum flux are not so large; $\mathscr{E}$ at $t=382 \pi$ is about $15 \%$ larger than 0.13 and $\mathscr{C}$ at $t=382 \pi$ is about $20 \%$ smaller than 0.076 .

\section{APPENDIX B: DERIVATION OF THE SECOND-ORDER SOLUTION}

As is well known, the determination of an asymptotic solution of weakly nonlinear acoustic wave is a singular perturbation problem, where a regular perturbation expansion yields a secular term, and accordingly, the expansion breaks down in the far field. The first-order solution (15) has been derived by using several singular perturbation methods ${ }^{27}-$ the method of renormalization, the method of multiple scales, the analytical method of characteristics, etc. The second-order solution (25) can be obtained by extending any of the above three to the second order; the extension is straightforward. In this appendix, however, we shall present the derivation of Eq. (25) from the exact solution of simple wave, Eq. (10), to clarify the relation between them.

To begin with, we shall introduce the parameter $\xi$ defined as

$$
\mu=\xi+M X(\mu),
$$

where $\mu$ is the parameter which appears in Eq. (10) and signifies the time when the characteristic $\mu=$ constant is issued from the oscillating plate. If $M$ is sufficiently small, $\mu$ is a singlevalued function of $\xi$, and hence $\xi=$ constant means the characteristic $\mu=$ constant. Then, $\xi$ denotes the value of the phase variable $y=t-x$ at the intersection point of the characteristic and locus of the plate in the $(y, t)$ plane.

Substituting Eq. (B1) into the first of Eq. (10) and expanding it in $M$ yields

$$
u=M X^{\prime}(\xi)+M^{2} X(\xi) X^{\prime \prime}(\xi)+O\left(M^{3}\right) .
$$

By using Eq. (B1), we can rewrite the second of Eq. (10) as

$$
\xi=y+\beta M(t-\mu) X^{\prime}(\mu) .
$$

Since $y=t-x$, the second of Eq. (10) is also transformed into

$$
\beta M(t-\mu)=\frac{\beta M x-\beta M^{2} X(\mu)}{1+\beta M X^{\prime}(\mu)} .
$$

Substituting Eq. (B4) into Eq. (B3) and expanding the resultant equation in $M$ with the help of Eq. (B1) gives

$$
\begin{aligned}
\xi= & y+\beta x X^{\prime}(\xi)+M^{2} \beta x X(\xi) X^{\prime \prime}(\xi) \\
& -M^{2} \beta^{2} x\left[X^{\prime}(\xi)\right]^{2}+O\left(M^{2}, M^{3} x\right)
\end{aligned}
$$

We thus obtain the approximate expressions for $u$ and for $\xi$, Eqs. (B2) and (B5). Clearly, the linear solution is given by $u=M X^{\prime}(\xi)$ and $\xi=y$. Neglecting the terms multiplied by $M^{2}$ in Eqs. (B2) and (B5) leads to the first-order solution (15). The second-order solution (25) is composed of Eq. (B2) and (B5).

${ }^{\mathrm{I}}$ Y. Inoue and T. Yano, "Propagation of strongly nonlinear plane waves," J. Acoust. Soc. Am. 94, 1632 (1993); "Propagation of acoustic shock waves of large amplitude," in Frontiers of Nonlinear Acoustics, edited by M. F. Hamilton and D. T. Blackstock (Elsevier, London, 1990), pp. 141146.

${ }^{2}$ The diffusivity of sound $\delta$ is defined as $\delta=\nu[(4 / 3)+(\zeta / \eta)$ $\left.+(\gamma-1) \kappa / \eta c_{p}\right]$, where $\nu=\eta / \rho_{0}$ is the kinematic viscosity, $\rho_{0}$ is the density in an initial undisturbed gas, $\eta$ is the viscosity, $\zeta$ is the bulk viscosity, $\gamma$ is the ratio of specific heats, $\kappa$ is the thermal conductivity, and $c_{p}$ is the specific heat for the ideal gas at constant pressure. See, M. J. Lighthill, "Viscosity effects in sound waves of finite amplitude," in Surveys in Mechanics, edited by G. K. Batchelor and R. M. Davies (Cambridge University Press, Cambridge, 1956), pp. 250-351.

${ }^{3}$ Strictly speaking, condition $\operatorname{Re} \gg 1$ signifies the limit as $\operatorname{Re} \rightarrow \infty$, mathematically.

${ }^{4}$ S. Osher and S. R. Chakravarthy, "Very high order accurate TVD schemes," in Oscillation Theory, Computation, and Methods of Compensated Compactness, edited by C. Dafermos, J. Ericksen, D. Kinderlehrer, and M. Slemrod (Springer, New York, 1986), pp. 229-274.

${ }^{5} \mathrm{~S}$. Osher and F. Solomon, "Upwind difference schemes for hyperbolic systems of conservation laws," Math. Comput. 38, 339 (1982).

${ }^{6}$ S. I. Soluyan and R. V. Khokhlov, "Propagation of acoustic waves of finite amplitude in a dissipative medium," Vestn. Moskov. Univ. Fiz. Astron. 3, 52 (1961).

${ }^{7}$ D. T. Blackstock, "Connection between the Fay and Fubini solutions for plane sound waves of finite amplitude." J. Acoust. Soc. Am. 39, 1019 (1966).

${ }^{8}$ O. V. Rudenko and S. I. Soluyan, Theoretical Foundations of Nonlinear Acoustics (Consultants Bureau, New York, 1977).

${ }^{9}$ D. G. Crighton, "Basic theoretical nonlinear acoustics," in Frontiers in Physical Acoustics, edited by D. Sette (North-Holland, Amsterdam, 1986), pp. 1-52.

${ }^{10} \mathrm{~T}$. Yano and Y. Inoue, "Numerical study of strongly nonlinear acoustic waves, shock waves, and streaming caused by a harmonically pulsating sphere," Phys. Fluids 6, 2831 (1994).

${ }^{11} \mathrm{~T}$. Yano and Y. Inoue, "Strongly nonlinear waves radiated by a circular piston," in Advances in Nonlinear Acoustics, edited by H. Hobæk (World Scientific, Singapore, 1993), pp. 583-588; "'Strongly nonlinear waves and streaming in the near field of a circular piston," J. Acoust. Soc. Am. (in press).

${ }^{12}$ M. J. Lighthill, "The energy distribution behind decaying shocks.-I. Plane waves," Philos. Mag. 41, 1101 (1950).

${ }^{13} \mathrm{~J}$. E. Phythian, "The energy distribution behind a decaying twodimensional shock," Q. J. Mech. Appl. Math. 5, 318 (1952).

${ }^{14}$ C. L. Morfey and V. W. Sparrow, "Plane compression front steepening in nonlinear media forms both a shock and a reflected wave,' J. Acoust. Soc. Am. 93, 3085 (1993).

${ }^{15}$ That is, we shall seek a weak solution of the system (4)-(6). See, J. Smoller, Shock Waves and Reaction-Diffusion Equations (Springer, New York, 1983), Chap. 15.

${ }^{16} \mathrm{R}$. Courant and K. O. Friedrichs, Supersonic Flow and Shock Waves (Springer, New York, 1948), Sec. 40.

${ }^{17} \mathrm{~S}$. Earnshaw, "On the mathematical theory of sound," Trans. R. Soc. London. 150, 133 (1860).

${ }^{18} \mathrm{D}$. T. Blackstock, "Propagation of plane sound waves of finite amplitude in nondissipative fluids," J. Acoust. Soc. Am. 34, 9 (1962).

${ }^{19}$ G. B. Whitham, Linear and Nonlinear Waves (Wiley, New York, 1974), Sec. 2.8 .

${ }^{20}$ L. D. Landau and E. M. Lifshitz, Fluid Mechanics, 2nd ed. (Pergamon, Oxford, 1987), Sec. 102, problem 1, p. 390. 
${ }^{21}$ According to the numerical result in Ref. $1, V_{S}$ is approximately proportional to $M$, and hence $\bar{\rho} u$ is approximately proportional to $M^{2}$.

${ }^{22} Y$. Inoue and T. Yano, "Propagation of strongly nonlinear plane $N$ waves," J. Fluid Mech. (submitted).

${ }^{23}$ See Ref. 19, Sec. 6.10.

${ }^{24}$ If $X^{\prime \prime}(t)$ has some minima in the period $2 \pi$, then the shocks of the same number may be formed in each wave cycle; see, for example, Y. Inoue and Y. Gotoh, "Formation of weak shock waves caused by a sphere pulsating at large amplitude and low frequency," J. Sound Vib. 146, 269 (1991). It is easy to extend Eq. (32) to such a situation.

${ }^{25}$ See Ref. 20, Sec. 101, problem 4, pp. 384-385. See also, T. Yano, "The mean pressure and density in a strongly nonlinear plane acoustic wave," J. Acoust. Soc. Am. 100 (in press).

${ }^{26}$ I. I. Glass, L. E. Heuckroth, and S. Molder, "'One-dimensional overtaking of a shock wave by a rarefaction wave," ARS J. 31, 1453 (1961)

${ }^{27}$ A. H. Nayfeh and A. Kluwick, "A comparison of three perturbation methods for nonlinear hyperbolic waves," J. Sound Vib. 48, 293 (1976). 